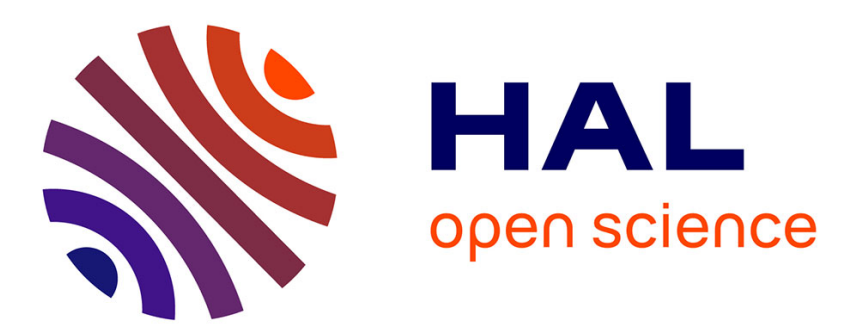

\title{
A tree-BLSTM-based recognition system for online handwritten mathematical expressions
}

\author{
Ting Zhang, Harold Mouchère, Christian Viard-Gaudin
}

\section{To cite this version:}

Ting Zhang, Harold Mouchère, Christian Viard-Gaudin. A tree-BLSTM-based recognition system for online handwritten mathematical expressions. Neural Computing and Applications, 2018, 10.1007/s00521-018-3817-2 . hal-01900412

\section{HAL Id: hal-01900412 \\ https://hal.science/hal-01900412}

Submitted on 29 Oct 2018

HAL is a multi-disciplinary open access archive for the deposit and dissemination of scientific research documents, whether they are published or not. The documents may come from teaching and research institutions in France or abroad, or from public or private research centers.
L'archive ouverte pluridisciplinaire HAL, est destinée au dépôt et à la diffusion de documents scientifiques de niveau recherche, publiés ou non, émanant des établissements d'enseignement et de recherche français ou étrangers, des laboratoires publics ou privés. 


\title{
A Tree-BLSTM based Recognition System for Online Handwritten Mathematical Expressions
}

\author{
Ting ZHANG · Harold MOUCHERE • \\ Christian VIARD-GAUDIN
}

Received: date / Accepted: date

\begin{abstract}
Long short- term memory networks (LSTM) achieves great success in temporal dependency modelling for chain-structured data, such as texts and speeches. An extension towards more complex data structures as encountered in $2 \mathrm{D}$ graphic languages is proposed in this work. Specifically, we address the problem of handwritten mathematical expression recognition, using a Treebased BLSTM architecture allowing the direct labelling of nodes (symbol) and edges (relationship) from a graph modelling the input strokes. One major difference with the traditional approaches is that there is no explicit segmentation, recognition and layout extraction steps but a unique trainable system that produces directly a Stroke Label Graph describing a mathematical expression. The proposed system, considering no grammar, achieves competitive results in online math expression recognition domain.
\end{abstract}

Keywords Mathematical expression recognition · tree-based BLSTM · local $\mathrm{CTC} \cdot$ online handwriting.

\section{Introduction}

A visual language is defined as any form of communication that relies on twoor three-dimensional graphics rather than simply (relatively) linear text [1]. Mathematical expressions (Figure 1), plans and musical notations are com-

Ting ZHANG

National Engineering Research Center for E-learning, Central China Normal University

E-mail: ting.zhang@mail.ccnu.edu.cn

Harold MOUCHERE

LS2N/IPI - UMR CNRS 6004, Université de Nantes, France

Christian VIARD-GAUDIN

LS2N/IPI - UMR CNRS 6004, Université de Nantes, France 


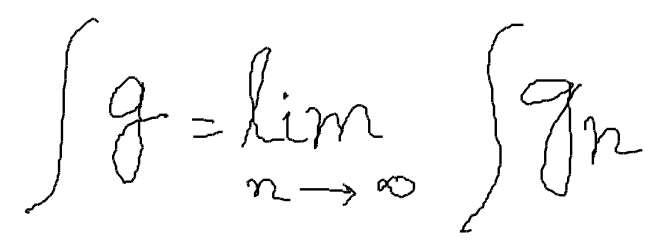

Fig. 1 Handwritten mathematical expression example

monly used cases of visual languages [2]. As an intuitive and easily (relatively) comprehensible knowledge representation model, mathematical expressions could help the dissemination of knowledge in some related domains and therefore are essential in scientific documents. Currently, common ways to input mathematical expressions into electronic devices include typesetting systems such as IATEX and mathematical editors such as the one embedded in MSWord. But these ways require that users could hold a large number of codes and syntactic rules, or handle troublesome manipulations with keyboards and mouses as interface. As another option, being able to input mathematical expressions by hand with a pen tablet, as we write them on paper, is a more efficient and direct mean to help the writing of scientific documents.

Handwritten mathematical expression recognition is an appealing topic in pattern recognition field since it exhibits a big research challenge and underpins many practical applications. Difficulties are related to the large set of symbols (more than 100) with many overlapping classes, and because of the 2 dimensional (2-D) structures between pairs of symbols (superscript, subscript, fraction etc.). With regard to the application, it offers an easy and direct way to input MEs into computers, and therefore improves productivity for scientific writers. We usually divide handwritten MEs into online and offline domains. In the offline domain, data is available as an image, while in the online domain it is a sequence of strokes, which are themselves sequences of points recorded along the pen trajectory. Compared to the offline ME, time information is available in online form. This paper will be focused on online handwritten ME recognition.

Generally, three tasks are involved in ME recognition [3,4]: (1) symbol segmentation, which consists in grouping strokes that belong to the same symbol; (2) symbol recognition, the task of labelling the symbols to assign each of them a symbol class; (3) structural analysis, its goal is to identify spatial relations between symbols and with the help of a grammar to produce a mathematical interpretation. The state of the art solutions are mainly grammar-driven solutions: a set of symbol hypotheses maybe generated and a structural analysis algorithm (grammar parsing usually) may select the best hypotheses while building the structure. However, these classical grammar-driven solutions, require not only a large amount of manual work for defining grammars, but also a high computational complexity for the grammar parsing process. Using a grammar is not mandatory; it can help finding a correct solution when working in a controlled environment. But the problem of fixing a grammar is 
context dependent, and less general than enabling the system to generate independently its own language model. As an alternative approach, we propose to explore a non grammar-driven solution for recognizing math expressions. This is the main goal of this work, we would like to propose new architectures for mathematical expression recognition with the idea of taking advantage of the recent advances in recurrent neural networks.

Advanced recurrent neural network - Long Short-Term Memory (LSTM) - achieved great success in temporal dependency modeling for chain-structured data, such as texts and speeches [5-7]. This success is due to LSTM's representational power and effectiveness at capturing long-term dependency in a sequence. Recently, research on LSTM has been pushed beyond sequential structures. It was extended to tree structures in $[8,9]$ and DAG (directed acyclic graph) structures in [10]. In this work, we put efforts on a similar task, generalizing the classical LSTM architecture to a tree network topology named tree-based BLSTM. Thereby, the new topology could handle tree-structured data as well as sequence-structured data.

In this work, we have been exploring online handwritten ME recognition from a new perspective, treating it as a problem of deriving a graph from the raw input, and then, extracting several trees which are embbeded in the graph and recognized them with a tree-based BLSTM. As known, it is possible to describe a ME using a Stroke Label Graph (SLG, refer to Section 2.2.2) in which nodes represent strokes whereas labels on the edges encode either segmentation or layout information. In $[11,12]$, the solution of building a graph by merging multiple $1 \mathrm{D}$ sequences of labels produced by a sequence labeller was proposed and explored. We extend these preliminary works by integrating multiple trees labelled by a tree labeller to recognize MEs.

In Section 2, the related works will be reviewed, including the state of the art of ME recognition and representation, and an overview of LSTM. Treebased BLSTM, as the base of our recognition system, is described in detail in Section 3. Afterwards, we introduce our tree-based BLSTM recognition system in Section 4 step by step, which is the main part of our study. At last, experiments and conclusion are covered in Sections 5 and 6 respectively.

\section{Related works}

\subsection{Mathematical expression recognition}

Research on the recognition of math notation began in the 1960s [13], and several research publications are available in the following thirty years [14-16]. Since the 90's, with the large developments of touch screen devices, this field has started to be active, producing amounts of research outcomes and gaining considerable attention from the research community. A number of surveys $[17-19,3,4]$ summarize the proposed techniques for math notation recognition. This research domain has been boosted by the Competition on Recognition of Handwritten Mathematical Expressions (CROHME) [4], which began as 
part of the International Conference on Document Analysis and Recognition (ICDAR) in 2011. It provides a platform for researchers to test their methods and compare them, and then facilitate the progress in this field. In this paper, the provided data and evaluation tools from CROHME will be used and results will be compared to participants.

As described already in Section 1, ME recognition involves three interdependent tasks [3]: (1) Symbol segmentation; (2) symbol recognition; (3) structural analysis. These three tasks can be solved sequentially or jointly. The proposed solutions can be roughly divided into sequential solutions and integrated solutions. In addition, with recent advances in deep learning, several end-to-end deep learning based systems were proposed for ME recognition. In the coming paragraphs, we introduce shortly these three types of proposed solutions for ME recognition.

Sequential solutions. In the early stages of the study, most of the proposed solutions [20-30] are sequential ones which treat the recognition problem as a two-step pipeline process; first symbol segmentation and classification, and then structural analysis. The task of structural analysis is performed on the basis of the symbol segmentation and classification result. Considerable works are done dedicated to each step. For segmentation, the proposed methods include minimum spanning tree (MST) based method [24], Bayesian framework [30], graph-based method [23,27] and so on. The symbol classifiers used consist of Nearest Neighbor, Hidden Markov Model, Multilayer Perceptron, Support Vector Machine, Recurrent neural networks and so on. For spatial relationship classification, the proposed features include symbol bounding box [13], relative size and position [31], and so on. The main drawback of these sequential methods is that the errors from symbol segmentation and classification will be propagated to structural analysis. In other words, symbol recognition and structural analysis are assumed as independent tasks in the sequential solutions. However, this assumption conflicts with the real case in which these three tasks are highly interdependent by nature. For instance, human beings recognize symbols with the help of structure, and vice versa.

Integrated solutions. Considering the natural mutual relationship between the three tasks, researchers mainly focus on integrated solutions recently, which performs the task of segmentation at the same time build the expression structure: a set of symbol hypotheses maybe generated and a structural analysis algorithm may select the best hypotheses while building the structure. The integrated solutions use contextual information (syntactic knowledge) to guide segmentation or recognition, preventing from producing invalid expressions like $[a+b)$. These approaches take into account contextual information generally with grammar (string grammar [32-36] and graph grammar $[37,38]$ ) parsing techniques, producing expressions conforming to the rules of a manually defined grammar. Either string or graph grammar parsing, both have a high time complexity. Instead of using grammar parsing technique, the new architectures proposed in this paper include contextual information with bidirectional long short-term memory which can access the content from both the future and the past in an unlimited range. 
End-to-end neural network based solutions. Inspired by recent advances in image caption generation, some end-to-end deep learning based systems were proposed for ME recognition [39,40]. These systems were developed from the attention-based encoder-decoder model which is now widely used for machine translation. They decompile an image directly into presentational markup language such as $\mathrm{AT}_{\mathrm{E}} \mathrm{X}$. However, considering we are given trace information in the online case, despite the final $\mathrm{AT}_{\mathrm{EX}}$ string, it is interesting to decide a label for each stroke. This information is not available now in end-to-end systems.

\subsection{Mathematical expression representation}

Structures can be depicted at three different levels: symbolic, object and primitive [41]. In the case of handwritten ME, the corresponding levels are expression, symbol and stroke.

In this section, we will first focus on symbol relation tree (SRT), one of the representation models for math expression at the symbol level. From the SRT, when going down to the stroke level, a stroke label graph (SLG) could be derived, which is the current official format to represent the ground-truth of handwritten math expressions and also for the recognition outputs in Competitions CROHME.

\subsubsection{Symbol level: Symbol relation (layout) tree}

When only a syntactic representation of a ME is required, operator trees based on symbol operators are adequate tools to describe the ME, but if layout is concerned, symbol relation (or layout) trees are more appropriate. Symbol layout tree represents the placement of symbols on baselines (writing lines), and the spatial arrangement of the baselines [3]. In SRT, nodes represent symbols, while labels on the edges indicate the relationships between symbols. For example, in Figure 2a, the first symbol '-' on the base line is the root of the tree; the symbol 'a' is Above '-' and the symbol 'c' is Below '-'. In Figure $2 \mathrm{~b}$, the symbol 'a' is the root; the symbol ' + ' is on the Right of 'a'.

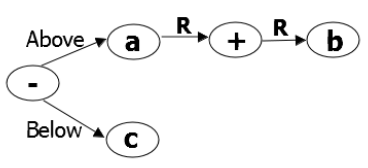

(a)

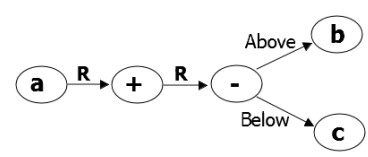

(b)

Fig. 2 The symbol relation tree (SRT) for (a) $\frac{a+b}{c}$, (b) $a+\frac{b}{c}$. ' $R$ ' refers to Right relationship

101 classes of symbols are present in CROHME data set, including digits, alphabets, operators and so on. Six spatial relationships are defined in the 
CROHME competition, they are: Right, Above, Below, Inside (for square root), Superscript, Subscript.

\subsubsection{Stroke level: Stroke label graph}

SRT represents math expression at the symbol level. If we go down at the stroke level, a stroke label graph (SLG) can be derived from the SRT. In SLG, nodes represent strokes, while labels on the edges encode either segmentation information or symbol relationships. Relationships are defined at the level of symbols, implying that all strokes (nodes) belonging to one symbol have the same input and output edges. Consider the simple expression $2+2$ written using four strokes (two strokes for ' + ') in Figure 3a. The corresponding SRT and SLG are shown in Figure 3b and Figure 3c respectively. As Figure 3c illustrates, nodes of SLG are labeled with the class of the corresponding symbol to which the stroke belongs. A dashed edge refers to segmentation information;

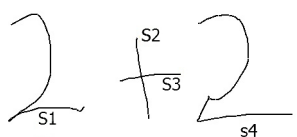

(a)

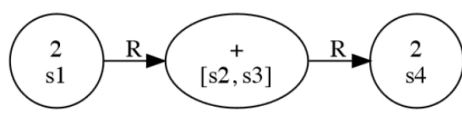

(b)

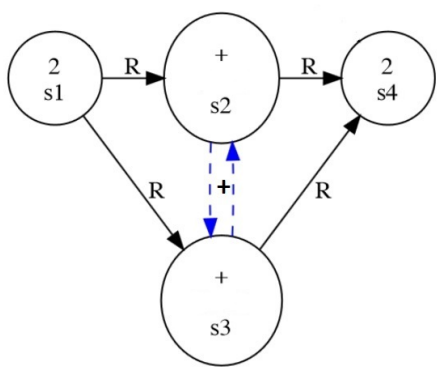

(c)

Fig. 3 (a) $2+2$ written with four strokes; (b) the symbol relation tree of $2+2$; (c) the SLG of $2+2$. The four strokes are indicated as s1, s2, s3, s4 in writing order. ' $R$ ' is for left-right relationship

it indicates that a pair of strokes belongs to the same symbol. In this case, the edge label is the same as the common symbol label. On the other hand, the non-dashed edges define spatial relationships between nodes and are labeled with one of the six different possible relationships between symbols. As a consequence, all strokes belonging to the same symbol are fully connected, nodes and edges sharing the same symbol label; when two symbols are in 
relation, all strokes from the source symbol are connected to all strokes from the target symbol by edges sharing the same relationship label.

Since CROHME 2013, SLG has been used to represent mathematical expressions [4]. As the official format to represent the ground-truth of handwritten math expressions and also for the recognition outputs, it allows detailed error analyses on stroke, symbol and expression levels. The recognition system that we propose works directly with the raw stroke inputs. It will produce a SLG directly comparable to the ground truth SLG. To that end, a label will be assigned to each stroke and to each pair of strokes involved in a symbol relation.

\subsection{Long Short-Term Memory Networks}

Recurrent neural networks (RNNs). RNNs can access contextual information and therefore are suitable for sequence labeling tasks [42]. We show an unfolded single-directional recurrent network in Figure 4, where each node at

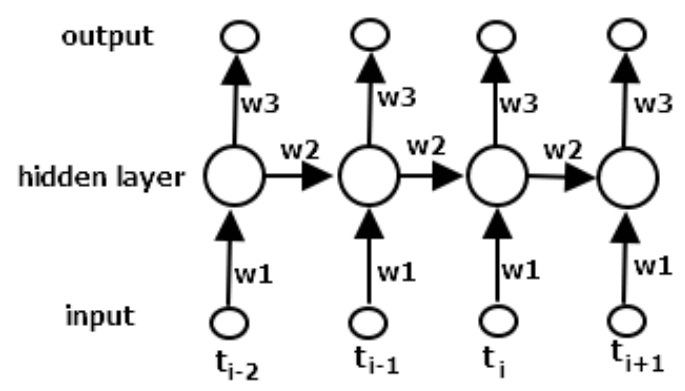

Fig. 4 An unfolded single-directional recurrent network

a single time-step represents a layer of network units. The network output at step $t_{i}$ depends on both the current input at step $t_{i}$ and the hidden state of $t_{i-1}$. The same weights $(w 1, w 2, w 3)$ are reused at every time-step.

LSTM. Unfortunately, with standard RNN architectures, the range of context that can be accessed is quite limited due to the vanishing gradient problem [43]. Long short-term memory (LSTM) [44] could address this problem by introducing a memory block which has the ability to preserve the state over long period of time. An LSTM network is similar to a standard RNN, except that the summation units in the hidden layer are replaced by memory blocks. Each block contains one or more self-connected memory cells and three multiplicative units (the input, output and forget gates). The three gates collect activation from inside and outside the block and control the activation of the cell via multiplications. The input and output gates multiply the input and output of the cell while the forget gate multiplies the cells previous state. 
The only output from the block to the rest of the network emanates from the output gate multiplication.

BLSTM. LSTM network processes the input sequence from past to future while Bidirectional LSTM [45], consisting of 2 separated LSTM layers, models the sequence from two opposite directions (past to future and future to past) in parallel. Both of 2 LSTM layers are connected to the same output layer. With this setup, complete long-term past and future context is available at each time step for the output layer.

Deep BLSTM. DBLSTM [46] can be created by stacking multiple BLSTM layers on top of each other in order to get higher level representation of the input data. The outputs of 2 opposite hidden layer at one level are concatenated and used as the input to the next level.

Non-chain-structured LSTM. A limitation of the classical LSTM network topology is that they only allow for sequential information propagation since the cell contains a single recurrent connection (modulated by a single forget gate) to its own previous value. Recently, research on LSTM has been beyond sequential structure. The one-dimensional LSTM was extended to $\mathrm{n}$ dimensions by introducing $\mathrm{n}$ recurrent connections (one for each of the cell's previous states along $\mathrm{n}$ dimensions) with $\mathrm{n}$ forget gates such that the new model could take into account the context from $n$ sources. It is named Multidimensional LSTM (MDLSTM) dedicated to the graph structure of an n-dimensional grid such as images [42]. MDLSTM model exhibits great performances on offline handwriting recognition tasks where the input is an image [47-51].

In [8], the basic LSTM architecture was extend to tree structures for improving semantic representations. Two extensions, the Child-sum Tree-LSTM and the $N$-ary Tree-LSTM, were proposed to allow for richer network topology where each unit is able to incorporate information from multiple child units. The Child-sum Tree-LSTM unit conditions its components on the sum of child hidden states. It is well-suited for trees with high branching factor or whose children are unordered. The $N$-ary Tree-LSTM can be used on tree structures where the branching factor is at most $N$ and where children are ordered. In parallel to the work in $[8,9]$ explored the similar idea and proposed $S$-LSTM model which provides a principled way of considering long-distance interaction over hierarchies, e.g., language or image parse structures. Furthermore, the DAG-structured LSTM was proposed for semantic compositionality in [10], possessing the ability to incorporate external semantics including noncompositional or holistically learned semantics.

Similar to the above mentioned works, we will extend the chain-structured BLSTM to tree-based BLSTM, and apply this new network model for online math expression recognition.

Connectionist temporal classification (CTC). With memory capability, RNNs are suitable for the sequence labelling tasks where the context is quite important. However, to apply this recurrent network for sequence labelling, there is a troublesome problem to be solved, being that at least a loss function should be defined for the training process. When using the typ- 
ical frame wise training method, the ground truth label of each time step is required to calculate the loss function, which implies that the training data should be segmented beforehand. The network is trained to make correct label prediction at each point. However, either the pre-segmentation or making label prediction at each point, both are large burdens to users or networks.

The technique of CTC was proposed to release these two burdens. It is specifically designed for sequence labeling problems where the alignment between the inputs and the target labels is unknown. By introducing an additional 'blank' class, CTC allows the network to make label predictions at some points instead of each point in the input sequence, so long as the overall sequence of character labels is correct. As described, CTC outputs directly sequences of labels, providing no information regarding the alignment between the inputs and the target labels.

Our proposal, building the SLG from the input handwritten ME, requires a label decision for each stroke and each stroke pair used in a symbol relation. To achieve this, we extend CTC to local CTC to relatively constrain the outputs and at the same time benefit from introducing an additional 'blank' class.

\section{The proposed Tree-based BLSTM}

This section will be focused on Tree-based BLSTM. Different with the tree structures depicted in $[8,9]$, we devote it to the kind of structures presented in Figure 5 where most nodes have only one next node. In fact, this kind of structure could be regarded as several chains with shared or overlapped segments. Traditional BLSTM process a sequence both from left to right and from right to left in order to access information coming from two directions. In our case, the tree will be processed from root to leaves and from leaves to root in order to visit all the surround context.

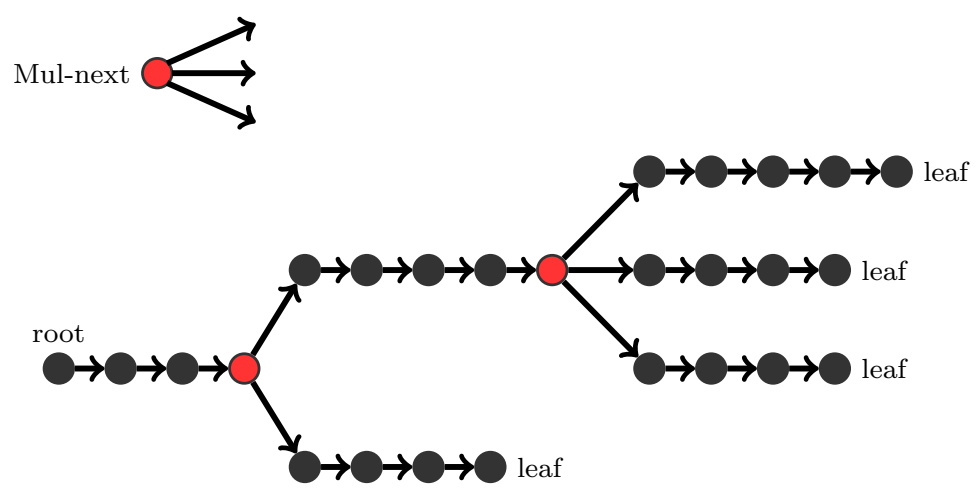

Fig. 5 A tree based structure for chains (from root to leaves)

From root to leaves. There are 2 special nodes (red) having more than one next node in Figure 5. We name them Mul-next node. The hidden states 
of Mul-next node will be propagated to its next nodes equally. The forward propagation of a Mul-next node is the same as for a chain LSTM node; with regard to the error propagation, the errors coming from all the next nodes will be summed up and propagated to Mul-next node.

From leaves to root. Suppose all the arrows in Figure 5 are reversed, we have the new structure which is actually beyond a tree in Figure 6 . The 2 red nodes are still special cases because they have more than one previous nodes. We call them Mul-previous nodes. The information from all the previous nodes will be summed up and propagated to the Mul-previous node; the error propagation is processed like for a typical LSTM node.

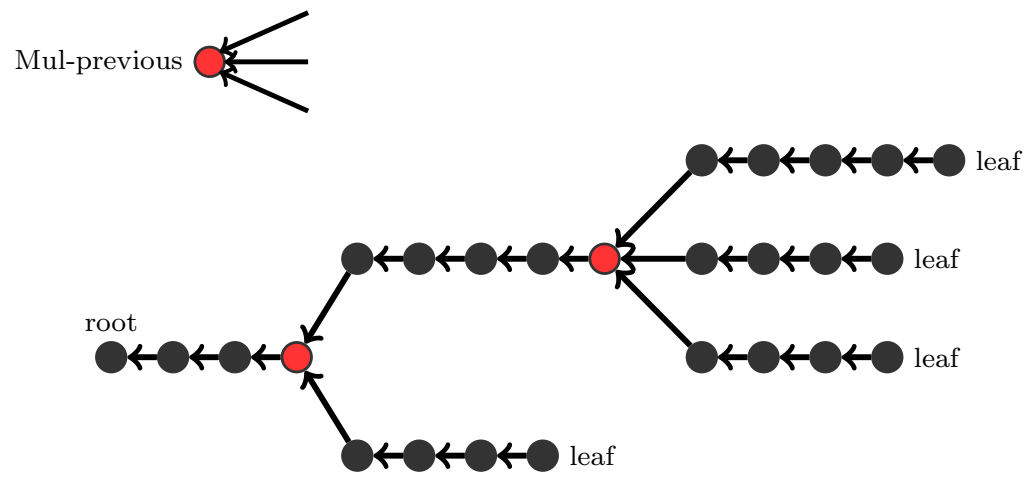

Fig. 6 A tree based structure for chains (from leaves to root)

We give the specific formulas below regarding to the forward propagation of Mul-previous node and the error back-propagation of Mul-next node. The same notations as in [42] are used here. The network input to unit $i$ at node $n$ is denoted $a_{i}^{n}$ and the activation of unit $i$ at node $n$ is $b_{i}^{n} \cdot w_{i j}$ is the weight of the connection from unit $i$ to unit $j$. Considering a network with $I$ input units, $K$ output units and $H$ hidden units, let the subscripts $\varsigma, \phi, \omega$ refer to the input, forget and output gate. The subscript $c$ refers to one of the $C$ cells. Thus, the peep-hole weights from cell $c$ to the input, forget, output gates can be denoted as $w_{c \varsigma}, w_{c \phi}, w_{c \omega} . s_{c}^{n}$ is the state of cell $c$ at node $n . f$ is the activation function of the gates, and $g$ and $h$ are respectively the cell input and output activation functions. $L$ is the loss function used for training.

We only give the equations for a single memory block. For multiple blocks the calculations are simply repeated for each block. Let $\operatorname{Pr}(n)$ denote the set of previous nodes of node $n$ and $\mathrm{Ne}(n)$ denote the set of next nodes. We highlight the different parts with box compared to the classical LSTM formulas. 


\section{The forward propagation of Mul-previous node}

\section{Input gate}

The input gate collects the activations from the current input $x_{i}^{n}$, the hidden unit activation $b_{h}^{p}$ of $\operatorname{Pr}(n)$ and the cell state $s_{c}^{p}$ of $\operatorname{Pr}(n) . f$ denote the gate activation function, usually the logistic sigmoid, so that the gate activations are between 0 (gate closed) and 1 (gate open).

$$
\begin{aligned}
a_{\varsigma}^{n}=\sum_{i=1}^{I} w_{i \varsigma} x_{i}^{n}+\sum_{h=1}^{H} w_{h \varsigma}\left|\sum_{p=1}^{|\operatorname{Pr}(n)|} b_{h}^{p}+\sum_{c=1}^{C} w_{c \varsigma}\right| \sum_{p=1}^{|\operatorname{Pr}(n)|} s_{c}^{p} \\
b_{\varsigma}^{n}=f\left(a_{\varsigma}^{n}\right)
\end{aligned}
$$

Forget gate

The forget gate works in the say way as the input gate.

$$
\begin{gathered}
a_{\phi}^{n}=\sum_{i=1}^{I} w_{i \phi} x_{i}^{n}+\sum_{h=1}^{H} w_{h \phi} \sum_{p=1}^{|\operatorname{Pr}(n)|} b_{h}^{p}+\sum_{c=1}^{C} w_{c \phi} \sum_{p=1}^{|\operatorname{Pr}(n)|} s_{c}^{p} \\
b_{\phi}^{n}=f\left(a_{\phi}^{n}\right)
\end{gathered}
$$

Cell

The input to the cell includes the current input $x_{i}^{n}$, the hidden unit activation of $\operatorname{Pr}(n) . g$ is the cell input activation function. The input gate controls the input of the cell via multiplication. Similarly, the forget gate decides on to which extent the cell could access the cell state $s_{c}^{p}$ of $\operatorname{Pr}(n)$.

$$
\begin{gathered}
a_{c}^{n}=\sum_{i=1}^{I} w_{i c} x_{i}^{n}+\sum_{h=1}^{H} w_{h c} \sum_{p=1}^{|\operatorname{Pr}(n)|} b_{h}^{p} \\
s_{c}^{n}=b_{\phi}^{n} \sum_{p=1}^{|\operatorname{Pr}(n)|} s_{c}^{p}+b_{\varsigma}^{n} g\left(a_{c}^{n}\right)
\end{gathered}
$$

\section{Output gate}

The output gate receives the activations from the current input $x_{i}^{n}$, the hidden unit activation of $\operatorname{Pr}(n)$ and the current cell state $s_{c}^{n}$.

$$
\begin{gathered}
a_{\omega}^{n}=\sum_{i=1}^{I} w_{i \omega} x_{i}^{n}+\sum_{h=1}^{H} w_{h \omega} \sum_{p=1}^{|\operatorname{Pr}(n)|} b_{h}^{p}+\sum_{c=1}^{C} w_{c \omega} s_{c}^{n} \\
b_{\omega}^{n}=f\left(a_{\omega}^{n}\right)
\end{gathered}
$$

Cell Output

The output gate controls the output of the cell via multiplication.

$$
b_{c}^{n}=b_{\omega}^{n} h\left(s_{c}^{n}\right)
$$


The error back-propagation of Mul-next node

Definitions

$$
\epsilon_{c}^{n}=\frac{\partial L}{\partial b_{c}^{n}} \quad \epsilon_{s}^{n}=\frac{\partial L}{\partial s_{c}^{n}} \quad \delta_{i}^{n}=\frac{\partial L}{\partial a_{i}^{n}}
$$

Cell output

$$
\epsilon_{c}^{n}=\sum_{k=1}^{K} w_{c k} \delta_{k}^{n}+\sum_{g=1}^{G} w_{c g} \sum_{e}^{|\mathrm{Ne}(n)|} \delta_{g}^{e}
$$

$\epsilon_{c}^{n}$ refers to the partial derivative of the loss function $L$ with respect to the output of cell $c$ at node $n$.

Output gate

$$
\delta_{w}^{n}=f^{\prime}\left(a_{w}^{n}\right) \sum_{c=1}^{C} h\left(s_{c}^{n}\right) \epsilon_{c}^{n}
$$

$\delta_{w}^{n}$ denotes the partial derivative of the loss function $L$ with respect to the input of output gate at node $n$.

\section{State}

$$
\begin{gathered}
\epsilon_{s}^{n}=b_{w}^{n} h^{\prime}\left(s_{c}^{n}\right) \epsilon_{c}^{n}+\sum_{e=1}^{|\mathrm{Ne}(n)|} b_{\phi}^{e} \sum_{e=1}^{|\mathrm{Ne}(n)|} \epsilon_{s}^{e} \\
+w_{c \varsigma} \mid \sum_{e=1}^{|\mathrm{Ne}(n)|} \delta_{\varsigma}^{e}+w_{c \phi} \sum_{e=1}^{|\mathrm{Ne}(n)|} \delta_{\phi}^{e}+w_{c \omega} \delta_{\omega}^{n}
\end{gathered}
$$

$\epsilon_{s}^{n}$ represents the partial derivative of the loss function $L$ with respect to the cell state at node $n$.

Cell

$$
\delta_{c}^{n}=b_{\varsigma}^{n} g^{\prime}\left(a_{c}^{n}\right) \epsilon_{s}^{n}
$$

$\delta_{c}^{n}$ is the partial derivative of the loss function $L$ with respect to the cell input at node $n$.

Forget gate

$$
\delta_{\phi}^{n}=f^{\prime}\left(a_{\phi}^{n}\right) \sum_{c=1}^{C} \sum_{p=1}^{|\operatorname{Pr}(n)|} s_{c}^{p} \epsilon_{s}^{n}
$$

$\delta_{\phi}^{n}$ denotes the partial derivative of the loss function $L$ with respect to the input of forget gate at node $n$.

Input gate

$$
\delta_{\varsigma}^{n}=f^{\prime}\left(a_{\varsigma}^{n}\right) \sum_{c=1}^{C} g\left(a_{c}^{n}\right) \epsilon_{s}^{n}
$$

$\delta_{\varsigma}^{n}$ refers to the partial derivative of the loss function $L$ with respect to the input of input gate at node $n$.

\section{The Tree-BLSTM based Recognition System}

In this section, we elaborate orderly each stage involved in the proposed system. The input data is available as a sequence of strokes $S$ from which we 
would like to obtain the correct SLG graph describing unambiguously the ME. Let $S=\left(s_{0}, \ldots, s_{n-1}\right)$, where we assume $s_{i}$ has been written before $s_{j}$ for $i<j$. Algorithm 1 presents the framework of the recognition system in an algorithm procedure format for clarity.

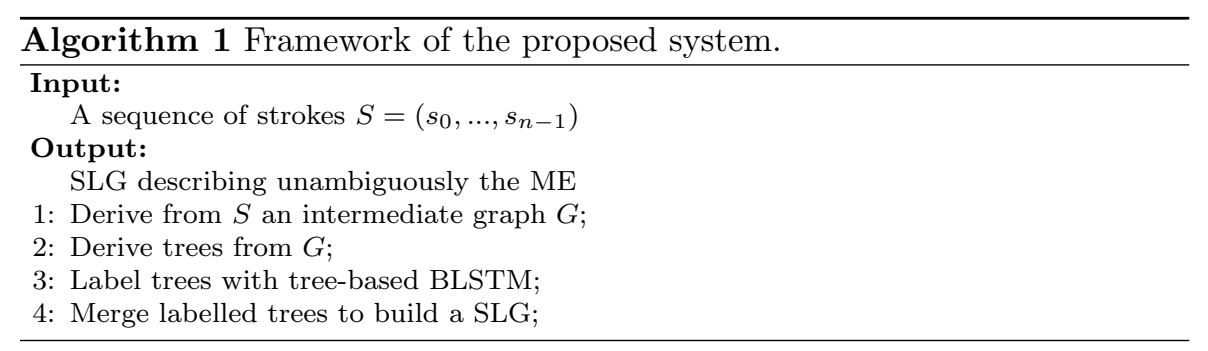

4.1 Derivation of an intermediate graph $G$

In a first step, we will derive from $S$ an intermediate graph $G$ where each node represents a stroke and edges are added according to several defined criteria. We provide several definitions related to the graph building first.

Definition $1 \mathrm{~A}$ stroke $s_{i}$ is considered visible from stroke $s_{j}$ if the straight line between their closest points does not intersect any other stroke $s_{k}$ of the ink.

For example, $s_{1}$ and $s_{3}$ can see each other because the straight line between their closest points does not intersect any other stroke of the ink, here $s_{2}$ or $s_{4}$ as shown in Figure 7. This definition is the same as the one used in [52].

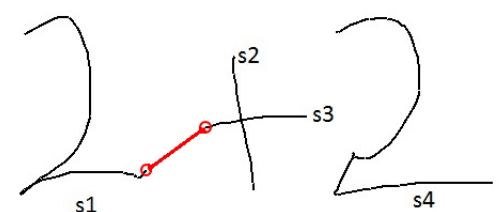

Fig. 7 Illustration of visibility between a pair of strokes. $s_{1}$ and $s_{3}$ are visible to each other

Definition 2 For each stroke $s_{i}$, we define 5 regions $(R 1, R 2, R 3, R 4, R 5$ shown in Figure 8) based on it. The center of the bounding box of stroke $s_{i}$ is taken as the reference point $(0,0)$.

The purpose of defining these 5 regions is to look for the potential Right, Inside, Above, Below, Supscript and Subscript relationships between strokes. 


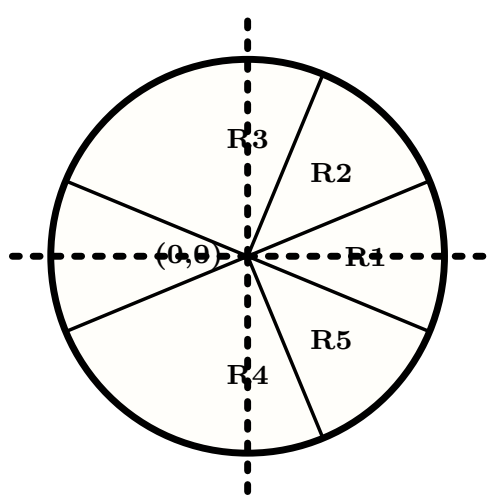

Fig. 8 Five regions for a stroke $s_{i}$. Point $(0,0)$ is the center of bounding box of $s_{i}$. The angle range of $R 1$ region is $\left[-\frac{\pi}{8}, \frac{\pi}{8}\right] ; R 2:\left(\frac{\pi}{8}, \frac{3 * \pi}{8}\right] ; R 3:\left(\frac{3 * \pi}{8}, \frac{7 * \pi}{8}\right] ; R 4:\left[-\frac{7 * \pi}{8},-\frac{3 * \pi}{8}\right)$; $R 5:\left[-\frac{3 * \pi}{8},-\frac{\pi}{8}\right)$

If the center of bounding box of $s_{j}$ is located in one of the five regions of stroke $s_{i}$, for example $R 1$ region, we say $s_{j}$ is in the $R 1$ direction of $s_{i}$. A wider searching range is defined for both $R 3$ and $R 4$ regions. That is because in some expressions like $\frac{a+b+c}{d+e+f}$, a larger searching range means more possibilities to catch the Above relationship from '-' to 'a' and the Below relationship from '-' to ' $d$ '.

Definition 3 Let $G$ be a directed graph in which each node corresponds to a stroke and edges are added according to the following criteria in succession.

We defined for each stroke $s_{i}(i$ from 0 to $n-2)$ :

- the set of strokes $S_{\text {int }}(i)=\left\{s_{\text {int } 1}, s_{\text {int } 2}, \ldots\right\}$, each element is from $\left\{s_{i+1}, \ldots, s_{n-1}\right\}$, and intersects $s_{i}$

For stroke $s_{i}(i$ from 0 to $n-1)$ :

- the set $S_{\text {vis }}(i)$ of the visible leftmost (considering the center of bounding box only) strokes in five directions respectively.

Edges from $s_{i}$ to the $S_{\text {int }}(i) \bigcup S_{\text {vis }}(i)$ will be added to $G$. Then, we check if the edge from $s_{i}$ to $s_{i+1}$ ( $i$ from 0 to $n-2$ ) exists in $G$. If not, this edge is added to $G$ to ensure that the path covering the sequence of strokes in the time order is included in $G$. Each edge is tagged depending on the specific criterion we used to find it before. Consequently, we have at most 7 types of edges (Intersection, $R 1, R 2, R 3, R 4, R 5$ and Time) in the graph. For those edges from $s_{i}$ to the $S_{\text {int }}(i) \cap S_{\text {vis }}(i)$, the type Inter section is assigned.

Figure 9 illustrates the process of deriving graph from raw input step by step using the example of $\frac{f}{a}=\frac{b}{f}$. First according to the 10 strokes in the raw input (Figure 9a), we create 10 nodes, one for each stroke; for each stroke, look for its intersecting strokes and add the corresponding edges labeled with Intersection between nodes (Figure 9b); proceeding to next step, for each 


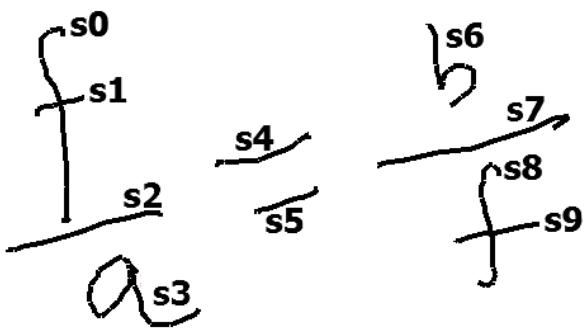

(a)

(s0) I (s1)

(s2)

(s4)

(s5)

(3)

(b) (s6)

(s7)

(s8) $\stackrel{\mathrm{I}}{\longrightarrow}$ (s9)

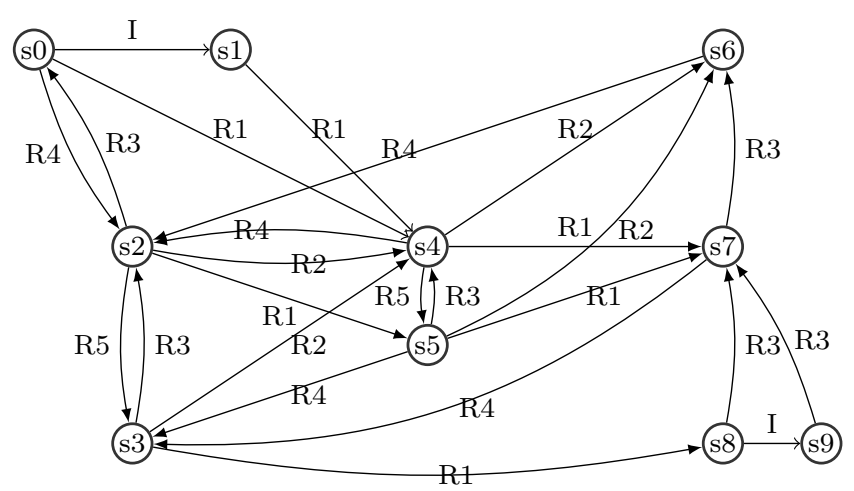

(c)

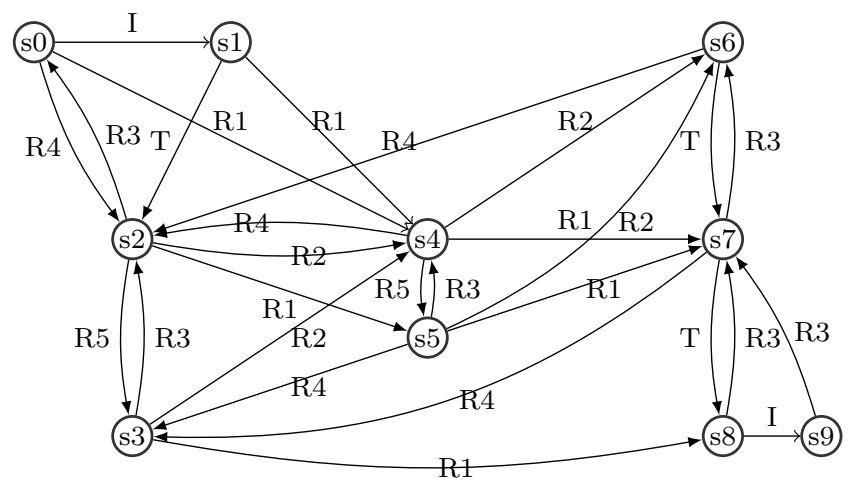

(d)

Fig. 9 (a) $\frac{f}{a}=\frac{b}{f}$ is written with 10 strokes; (b) add Intersection edges; (c) add $R 1, R 2, R 3, R 4, R 5$ edges; (d) add Time edges. I : Intersection, $T:$ Time 
stroke, look for its the visible rightmost strokes in five directions respectively and add the corresponding edges labeled as one of $R 1, R 2, R 3, R 4, R 5$ between nodes if the edges do not exist in the graph (Figure 9c); finally, check if the edge from $s_{i}$ to $s_{i+1}$ ( $i$ from 0 to $n-2$ ) exists in $G$ and if not, add this edge to $G$ labeled as Time to ensure that the path covering the sequence of strokes in the time order is included in $G$ (Figure 9d).

\subsection{Derivation of trees from $G$}

As described before in Section 4.1, we derive a graph from the raw input considering the temporal and spatial information. Then we will try to label nodes and edges of $G$ correctly in order to build a SLG finally. The solution proposed in this work is to derive multiple trees from $G$, then recognize the trees using the tree-based BLSTM model.

There exists different strategies to derive trees from $G$. In any of the cases, a start node should be selected first. We take the leftmost (considering the leftmost point in a stroke) stroke as the starter. From the starting node, we traverse the graph with the Depth-First Search algorithm. Each node should be visited only once. When there are more than one edge outputting from one node, the visiting order will follow the sequence of (Intersection, $R 1, R 3, R 4, R 2, R 5$, Time). With this strategy, a tree is derived to which we give the name Tree-Left-R1. It is dedicated to catch $R 1$ relationship. Figure 10 illustrates the derived TreeLeft-R1 for the ME $\frac{f}{a}=\frac{b}{f}$ written with 10 strokes. The Intersection edge

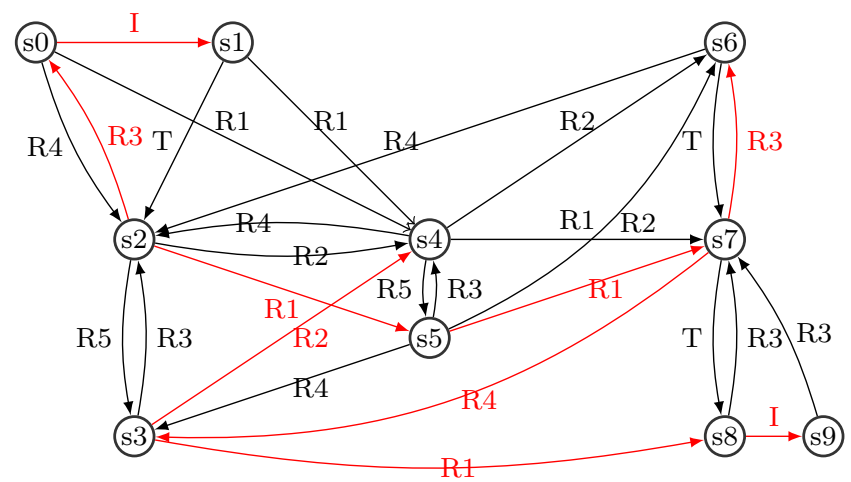

Fig. 10 The derived graph of the handwritten ME $\frac{f}{a}=\frac{b}{f}$ shown in Figure 9a. Tree-Left-R1 is highlighted with red color. $s 2$ is the root of it. $I$ : Intersection, $T:$ Time

is on the top of list, and it is because we assume that a pair of intersecting strokes belong to a single symbol. In Figure 10, Tree-Left-R1 is depicted in red with the root in $s 2$. Note that in this case, all the nodes are accessible from the start node $s 2$. However, as $G$ is a directed graph, some nodes are not reachable from one starter in some cases. Therefore, we consider deriving 
trees from different starters. Besides the leftmost stroke, it is interesting to derive trees from the first input stroke $s 0$ since sometimes users start writing an expression from its root. Note that in some cases, the leftmost stroke and stroke $s 0$ could be the same one. We replace the left-most stroke with stroke $s 0$ and keep the same strategy to derive the tree. The new tree is named as Tree-0-R1.

Finally, if $s 0$ is taken as the starting point and time order is considered first, a special tree is obtained which we call Tree-Time. Tree-Time is proposed with the aim of having a good cover of segmentation edges since users usually write a multi-stroke symbol continuously. As a matter of fact, it is a chain structure. Tree-Time is defined by $s 0 \rightarrow s 1 \rightarrow s 2 \rightarrow s 3 \ldots \rightarrow s 9$ for the expression in Figure 10. Table 1 offers a clear summary of the 3 derived trees from the graph. The experiment results presented in Section 5.2.2 prove the effectiveness of the method for deriving trees.

Table 1 The different types of derived trees

\begin{tabular}{|c|c|c|c|}
\hline Type & Root & Traverse algorithm & Visiting order \\
\hline Tree-Left-R1 & the leftmost stroke & Depth-First Search & (Intersection, $R 1, R 3, R 4, R 2, R 5$, Time $)$ \\
\hline Tree-0-R1 & $s 0$ & Depth-First Search & (Intersection, $R 1, R 3, R 4, R 2, R 5$, Time $)$ \\
\hline Tree-Time & $s 0$ & Depth-First Search & only the time order \\
\hline
\end{tabular}

\subsection{Feed the inputs of the Tree-based BLSTM}

In section 4.2 , we derived trees from the intermediate graph. Nodes of the tree represent visible strokes and edges denote the relationships between pairs of strokes. We would like to label each node and edge correctly with the Treebased BLSTM model, aiming to build a complete SLG finally. To realize this, the first step is to feed the derived tree into the Tree-based BLSTM model.

The solution is to go from the previous trees defined at the stroke level down to a tree at the point level, points being the raw information that are recorded along the pen trajectory in the on-line signal. To be independent from the different writing speeds, an additional re-sampling process should be carried out with a fixed spatial step. In the considered trees, nodes, which represent strokes, are re-sampled with a fixed spatial step, and the same holds for edges by considering the straight lines in the air between the last point and the first point of a pair of strokes that are connected in the tree. This is illustrated in Figure 11, where the re-sampled points are displayed inside the nodes (on-paper points for node) and above the edges (in-air points for edge). Since this tree will be processed by the BLSTM network, we need for the training stage to assign it a corresponding ground-truth. We derive it from the SLG by using the corresponding symbol label of the strokes (nodes) for the on-paper points and the corresponding symbol or relationship label for the in-air points (edges) when this edge exists in the SLG. When an 


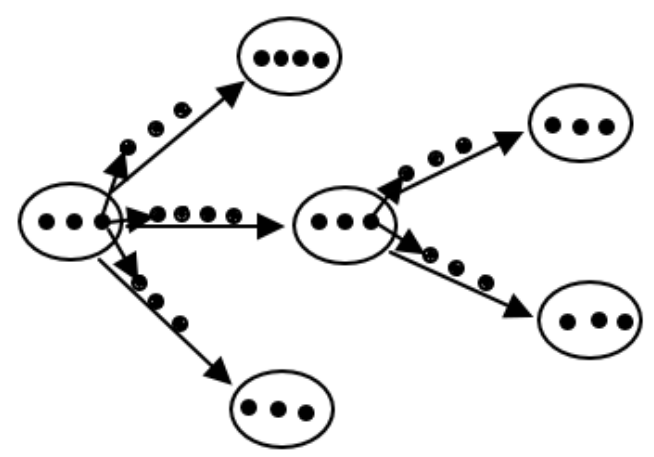

Fig. 11 A re-sampled tree. The small arrows between points provide the directions of information flows. With regard to the sequence of points inside one node or edge, most of small arrows are omitted

edge of the tree does not exist in the SLG, the label NoRelation noted '.' will be used. In this way, an edge in the graph which was originally denoted with a $I, R i(i=1 \ldots 5)$ or $T$ relation will be assigned with one of the 7 labels: (Right, Above, Below, Inside, Superscript, Subscript,_-) or a symbol label when the two strokes are belonging to the same symbol. Totally, for the ground truth, we have 108 classes(101 symbol classes +6 relationships + NoRelation).

The number of re-sampling points depends on the scale of the strokes with regard to the full expression scale. For each node or edge, we re-sample with $10 \times l / d$ points. Here, $l$ refers to the length of a visible stroke or a straight line connecting 2 strokes and $d$ refers to the average diagonal of the bounding boxes of all the strokes in an expression. Subsequently, for every point $p(x, y)$ we compute 5 features $[\sin \theta, \cos \theta, \sin \phi, \cos \phi, \operatorname{PenUD}]$. The full description of the features can be found in [12].

\subsection{Training process}

Figure 12 illustrates a tree-based BLSTM network with one hidden level. To provide a clear view, we only draw the full network on a short sequence (red) instead of a whole tree. Globally, the data structure we are dealing with is a tree; locally, it consists of several short sequences. For example, the tree presented in Figure 12 has 6 short sequences one of which is highlighted with red color. The system processes each node or edge (which is a short sequence in fact) separately but following the order with which the correct propagation of activation or errors could be ensured.

The training process of a short sequence (the red one in Figure 12 for example) is similar to the classical BLSTM model except that some information from adjacent short sequences should be taken into account. In the classical BLSTM case, the incoming activation or error of a short sequence is initialized as 0 . 


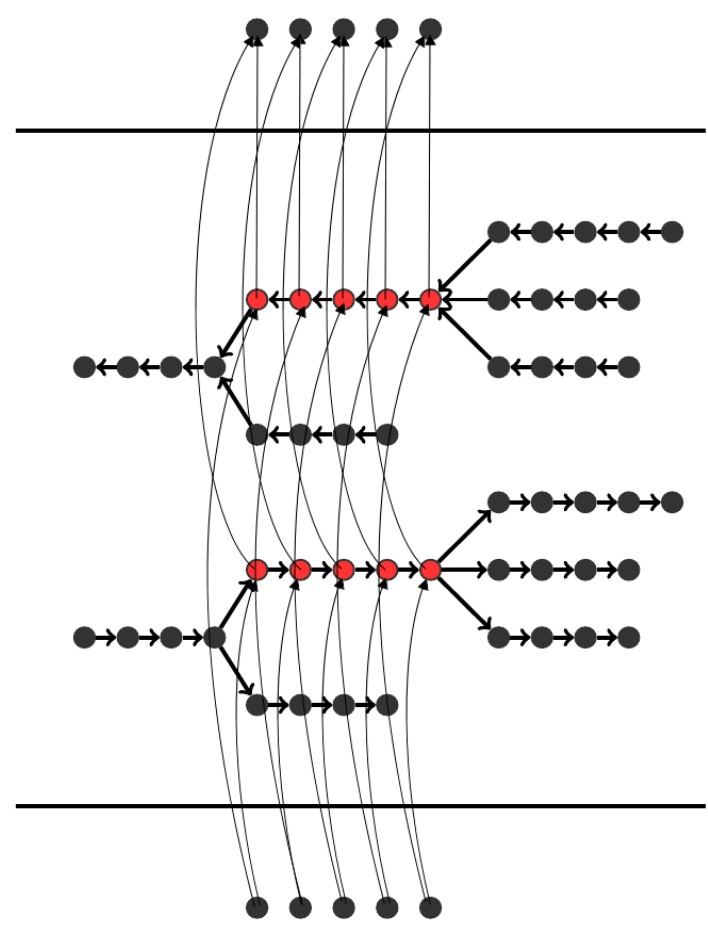

Output layer

Hidden layer ( from leaves to root)

Hidden layer ( from root to leaves)

Fig. 12 A tree-based BLSTM network with one hidden level. We only draw the full connection on one short sequence (red) for a clear view

Forward pass. Here, when proceeding with the forward pass from the input layer to the output layer, for the hidden layer (from root to leaves), we need to consider the coming information from the root direction and for the hidden layer (from leaves to root), we need to consider the coming information from the leaves direction. Obviously, no matter which kind of order for processing sequence we are following, it is not possible to have the information from both directions in one run. Thus another stage which we call pre-computation is required. The pre-computation stage has two runs: (1) From the input layer to the hidden layer (from root to leaves), we process the short sequence consisting of the root point first and then the next sequences. In this run, each sequence in the tree stores the activation from the root direction. (2) From the input layer to the hidden layer (from leaves to root), we process the short sequences consisting of the leaf point first and then the next sequences. In this run, each sequence in the tree sums and stores the activation from the leaf direction. After pre-computation stage, the information from both directions are available to each sequence thus the forward pass from input to output is straightforward. 
Error propagation. The backward pass of tree-based BLSTM network has 2 parallel propagation paths: (1) one is from the output layer to hidden layer (from root to leaves), then to the input layer; (2) the other one is from the output layer to hidden layer (from leaves to root), then to the input layer. As these 2 propagation are independent, no pre-computation stage is needed here. For propagation (1), we process the short sequences consisting of the leaf point first and then the next sequences. For propagation (2), we process the short sequence consisting of the root point first and then the next sequences. Note that when there are several hidden levels in the network, a pre-computation stage is required also for error propagation.

Loss function. It is known that BLSTM and CTC stage have better performance when a "blank" label is introduced during the training process [53], so that decision can be made only at some point in the input sequence. One of the characteristics of CTC is that it does not provide the alignment between the input and output, just the overall sequence of labels. As we need to assign each stroke a label to build a SLG, a relatively precise alignment between the input and output is preferred. Thus, a local CTC algorithm is used in this work aiming to limit the label into the corresponding stroke.

Inside each short sequence, or we can say each node or edge, a local CTC loss function is easy to be computed from the output probabilities related to this short sequence. The total CTC loss function of a tree is defined as the sum of all local CTC loss functions regarding to all the short sequences in this tree.

Since each short sequence has one label, the possible labels of the points in one short sequence are shown in Figure 13. For example, suppose character

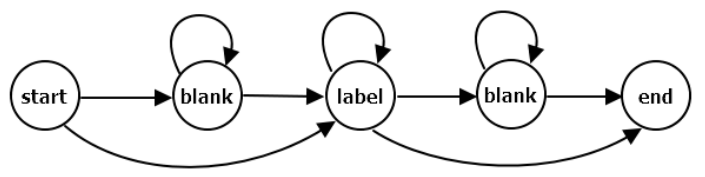

Fig. 13 The possible labels of points in one short sequence

$c$ is written with one stroke and 3 points are re-sampled from the stroke. The possible labels of these points can be $c c c, c c-, c--,--c,-c c$ and $-c-$ ('-' denotes 'blank'). More generally, the number of possible label sequences is $n *(n+1) / 2$ ( $n$ is the number of points), which is actually 6 with the proposed example.

Given the tree input represented as $X$ consisting of $N$ short sequences, each short sequence could be denoted as $X_{i}, i=1, \ldots, N$ with the ground truth label $l_{i}$ and the length $T_{i} . l_{i}^{\prime}$ represents the label sequence with blanks added to the beginning and the end of $l_{i}$, i.e. $l_{i}^{\prime}=\left(\right.$ blank,$l_{i}$, blank $)$ of length 3 . The forward variable $\alpha_{i}(t, u)$ denotes the summed probability of all length $t$ paths that are mapped by $F$ onto the length $u / 2$ prefix of $l_{i}$, where $u$ is from 1 to 3 and $t$ is from 1 to $T_{i}$. The mapping function $F$ is defined as first removing the repeated 
labels and then the blanks (-) from the paths. For example considering an short sequence of length 6 , two possible paths could be $--a a a-,---a a-$. The mapping function works like: $F(--a a a-)=F(---a a-)=a$.

Similarly, the backward variable $\beta_{i}(t, u)$ denotes the summed probabilities of all paths starting at $t+1$ that complete $l_{i}$ when appended to any path contributing to $\alpha_{i}(t, u)$. Figure 14 demonstrates the CTC forward-backward algorithm limited in one stroke.

li

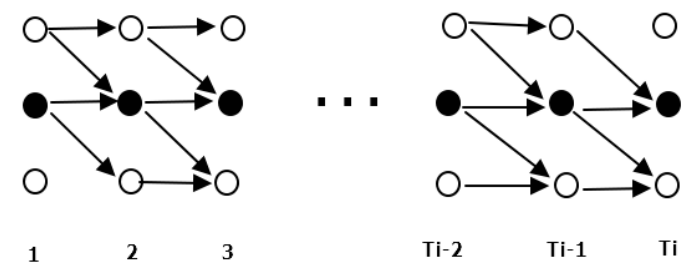

Fig. 14 CTC forward-backward algorithm in one stroke $X_{i}$. Black circle represents label $l_{i}$ and white circle represents blank. Arrows signify allowed transitions. Forward variables are updated in the direction of the arrows, and backward variables are updated in the reverse direction

With the CTC forward-backward algorithm (referring to [42] for details), we can compute the $\alpha_{i}(t, u)$ and $\beta_{i}(t, u)$ for each point $t$ and each allowed positions $u$ at point $t$. The local CTC loss function $L\left(X_{i}, l_{i}\right)$ is defined as the negative log probability of correctly labeling the short sequence $X_{i}$ :

$$
L\left(X_{i}, l_{i}\right)=-\ln p\left(l_{i} \mid X_{i}\right)
$$

According to the Equation (7.26) in [42], we can rewrite $L\left(X_{i}, l_{i}\right)$ as:

$$
L\left(X_{i}, l_{i}\right)=-\ln \sum_{u=1}^{3} \alpha_{i}(t, u) \beta_{i}(t, u)
$$

Then the errors will be back propagated to the output layer, then the hidden layer, finally to the entire network. The weights in the network will be updated after each entire tree structure is processed.

The CTC loss function of a entire tree structure is defined as the sum of the errors with regards to all the short sequences in this tree:

$$
L(X, l)=\sum_{i=1}^{N} L\left(X_{i}, l_{i}\right)
$$

This formula is used for evaluating the performance of the network, and therefore could be as the metric to decide the training process stops or not. 
4.5 Recognition process

As mentioned, the system treats each node or edge as a short sequence. A simple decoding method is adopted here. We choose for each node or edge the label which has the highest cumulative probability over the short sequence. Suppose that $y_{i}^{j}$ is the probability of outputting the $i$ label at the $j$ point. The probability of outputting the $i$ label can be computed as $P_{i}=\sum_{j=1}^{s} y_{i}^{j}$, where $s$ is the number of points in a short sequence. The label with the highest probability is assigned to this short sequence.

\subsection{Post process}

Several trees regarding to one expression will be merged to build a SLG after labeling. Besides the merging strategy, in this section we consider several structural constraints when building the SLG. Generally, 5 steps are included in post process:

(1) Merge trees. Each node or edge belongs at least to one tree, but possibly to several trees. Hence, several recognition results can be available for a single node or edge. We take an intuitive and simple way to deal with the problem of multiple results, choosing the one with the highest probability.

(2) Symbol segmentation. We look for the symbols using connected component analysis: a connected component where nodes and edges have the same label is a symbol.

(3) Relationships. We solve two possible kinds of conflicts in this step. (a) Perhaps between two symbols, there exists edges in both directions. Then, in each direction, we choose the label having the maximum probability. If the labels in two direction are both one of (Right, Above, Below, Inside, Superscript, Subscript) as illustrated in Figure 15a, we also choose the one having the larger probability. (b) Another type of conflict could be the case illustrated in Figure 15b where one symbol has two (or more) input relationships (one of 6 relationships). As introduced in Section 2.2.1, there is at most one input relationship for each node (symbol) in SRT. Therefore, when one symbol has two (or more) input relationships, we choose for it the one having the maximum probability.

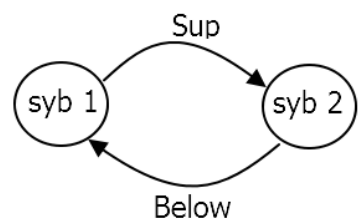

(a)

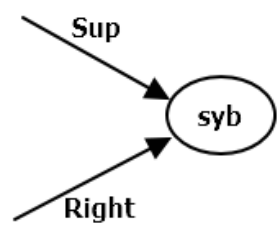

(b)

Fig. 15 Possible relationship conflicts existing in merging results 
(4) Make connected SRT. As SRT is a connected tree (this is a structural constraint, not a language specific constraint), there should be one root node and one or multiple leaf nodes. Each node has only one input edge, except the root node. After performing the first three steps, we still have the possibility to output a SRT containing several root nodes, in other words, being a forest instead of a tree. To address this type of error, we take a hard decision but quite simple: for each root $\mathbf{r}$ (except the one inputted earliest), add a Right edge to $\mathbf{r}$ from the leaf being the one nearest to $\mathbf{r}$ considering input time. We choose Right since it appears most in math expressions based on the statistics. (5) Add edges. According to the rule that all strokes in a symbol have the same input and output edges and that double-direction edges represent the segments, some missing edges can be completed automatically.

\subsection{Time complexity}

In this section, we analyse the time complexity of our Tree-BLSTM based recognition system and compare it with other grammar-driven solutions. As explained before, the proposed system consists of several sequential steps for each of which we will list the time complexity individually. First of all, the time complexity of the algorithm for building an intermediate graph is $O\left(n^{2}\right)$. Then to derive trees, the Depth-First Search algorithm requires $O(n+e)$ time where $e=7 n$ as there are at most $7 n$ edges in the intermediate graph. For activating the BLSTM, it requires $O(n)$ for nodes and $O(n)$ for edges (we are in a tree). The same time complexity holds for the recognition process. Finally, different operations in post process take linear time also. Therefore, the time complexity of our system is $O\left(n^{2}\right)$ in fact. Now, we consider the grammar-driven solutions. The time complexity of grammar parsing algorithm is $O\left(n^{3}|p|\right)$ at least where $|p|$ denotes the number of production rules. For example, it is $O\left(n^{3} \log (n)|p|\right)$ for the system proposed in [35]. Thus, we have a lower time complexity, $O\left(n^{2}\right)$ VS $O\left(n^{3}|p|\right)$ (at least).

\section{Experiments}

Data sets The complete data set from CROHME 2014 is used, 8834 expressions for training and 983 expressions for test. We extract randomly $10 \%$ of the 8834 expressions of the training set as a validation set. To get more recent comparison with the state of the art, we have also use the last CROHME 2016 data set to evaluate the best configuration. The training data set remains the same as CROHME 2014. However, 1147 new expressions are included in CROHME 2016 test data set.

Setup We developed the tree-based BLSTM recognition system with the RNNLIB library ${ }^{1}$. Several types of configurations are included in this paper:

1 Graves A. RNNLIB: A recurrent neural network library for sequence learning problems. http://sourceforge.net/projects/rnnl/. 
Network (i), (ii), (iii), (iv). The first one consists of one bidirectional hidden level (two opposite LSTM layers of 100 cells). This configuration has obtained good results in both handwritten text recognition [5] and handwritten math symbol classification [6,54]. Network (ii) is a deep structure with two bidirectional hidden levels, each containing two opposite LSTM layers of 100 cells. Network (iii) and Network (iv) have 3 bidirectional hidden levels and 4 respectively. The setup about the input layer and output layer remains the same. The size of the input layer is 5 ( 5 features); the size of the output layer is 109 $(101$ symbol classes +6 relationships + NoRelation + blank $)$.

With the Label Graph Evaluation library (LgEval) [55], the recognition results can be evaluated on symbol level and on expression level. We introduce several evaluation criteria: symbol segmentation (Segments), refers to a symbol that is correctly segmented whatever the label is; symbol segmentation and recognition (Seg+Class), refers to a symbol that is segmented and classified correctly; spatial relationship classification (Tree Rels.), a correct spatial relationship between two symbols requires that both symbols are correctly segmented and with the correct relationship label.

\subsection{Experiment 1}

Due to the deep structure, DBLSTM could generate higher level representation of the input data. In this experiment, we would like to see the effects of the depth of the network on the recognition results. And then according to the results, we choose the proper network configurations for the task. For each expression, the chain structure Tree-Time is derived to train the classifier.

The evaluation results on symbol level and global expression level are presented in Tables 2 and 3 respectively. From the tables, we can conclude that

Table 2 The symbol level evaluation results on CROHME 2014 test set with Tree-Time only

\begin{tabular}{|c|cc|cc|cc|}
\hline System & \multicolumn{2}{|c|}{ Segments (\%) } & \multicolumn{2}{|c|}{ Seg + Class (\%) } & \multicolumn{2}{c|}{ Tree Rels. (\%) } \\
\hline & Rec. & Prec. & Rec. & Prec. & Rec. & Prec. \\
\hline i, Tree-Time & 92.93 & 84.82 & 84.12 & 76.78 & 60.70 & 76.19 \\
\hline ii, Tree-Time & 95.10 & 90.47 & 87.53 & 83.27 & 65.06 & 83.18 \\
\hline iii, Tree-Time & 95.43 & 91.13 & 88.26 & 84.28 & 65.45 & 83.57 \\
\hline iv, Tree-Time & 95.57 & 91.21 & 87.81 & 83.80 & 65.98 & 82.85 \\
\hline
\end{tabular}

Table 3 The expression level evaluation results on CROHME 2014 test set with Tree-Time only

\begin{tabular}{|c|c|c|c|c|}
\hline System & correct (\%) & $\leq 1$ error & $\leq 2$ errors & $\leq 3$ errors \\
\hline i, Tree-Time & 12.41 & 20.24 & 26.14 & 30.93 \\
\hline ii, Tree-Time & 16.09 & 25.46 & 32.28 & 37.27 \\
\hline iii, Tree-Time & 16.80 & 25.56 & 32.89 & 38.09 \\
\hline iv, Tree-Time & 16.19 & 25.97 & 33.20 & 38.09 \\
\hline
\end{tabular}


as the network turns to be deeper, the recognition rate first increases and then stays at a relatively stable level. There is a large increase from Network (i) to Network (ii), a slight increase from Network (ii) to Network (iii) and no improvement from Network (iii) to Network (iv). These results show that 3 bidirectional hidden levels in the network is a proper option for the task in this work. Network with depth larger than 3 bring no improvement but higher computational complexity. Thus, for the coming experiments we will not take into account Network (iv) any more.

\subsection{Experiment 2}

In this section, we carry out experiments merging several trees to improve the coverage of the graph. As a first try, we derive only 3 trees, Tree-Time, Tree-Left- $R 1$ and Tree-0-R1 for each expression to train the classifiers separately. With regards to each tree, we consider 3 network configurations, being Network (i), Network (ii), Network (iii). Thus, we have 9 classifiers totally in this section. After training, we use these 9 classifiers to label the relevant trees and finally merge them to build a valid SLG. We merge the 3 trees labeled by the corresponding 3 classifiers which have the same network configuration to obtain the systems (i, Merge3), (ii, Merge3), (iii, Merge3). Then we merge the 3 trees labelled by all these 9 classifiers to obtain the system Merge 9 .

\subsubsection{Intermediate graph evaluation}

In Section 4.1, we described the method for deriving an intermediate graph from a sequence of strokes. This method should be evaluated to show its effectiveness. In [56], $\mathrm{Hu}$ evaluates the graph representation model by comparing the edges of the graph with ground truth edges at the stroke level where the recall and precision rates are considered both. We would like to take a similar but more intuitive method, detecting the missing and unnecessary relationships in the graph, which is at the symbol level. In order to evaluate if the selected trees will allow to recognize the symbol, we skip the recognition step in the process and label nodes and edges using the ground truth. The obtained graphs are then evaluated, with the standard with CROHME tool LgEval [55]. The symbols correctly recognised are those which could be recognized in the case of a perfect classification of each node and edge. Unrecognised symbol are due to missing edges. Obviously, a complete graph always leads to a perfect recognition.

Table 4 present the evaluation results of the intermediate graph on CROHME 2014 test set at the symbol level. As shown, the recall rate and the precision rate of segmentation task are almost $100 \%$, telling that the intermediate graph catches almost all the segmentation edges in the ground truth SLG. With regard to relationship recognition task, the graph catches $93.99 \%$ spatial relationships in expressions, missing around 6\%. Furthermore, as shown by the precision rate of the tree relations, there are in the graph more than half of 
the spatial relationships which are unnecessary. These unnecessary edges are expected to be labelled as NoRelation by the classifiers later.

Table 4 Intermediate graph evaluation results at the symbol level on CROHME 2014 test set (provided the ground truth labels of nodes and edges)

\begin{tabular}{|c|cc|cc|cc|}
\hline Model & \multicolumn{2}{|c|}{ Segments (\%) } & \multicolumn{2}{|c|}{ Seg + Class (\%) } & \multicolumn{2}{c|}{ Tree Rels. (\%) } \\
\hline & Rec. & Prec. & Rec. & Prec. & Rec. & Prec. \\
\hline Graph $G$ & 99.97 & 99.93 & 99.96 & 99.92 & 93.99 & 43.51 \\
\hline
\end{tabular}

\subsubsection{Derived trees evaluation}

As mentioned in the beginning of Section 5.2, we derive 3 trees, Tree-Time, Tree-Left-R1 and Tree-0-R1 for each expression to train the classifiers separately. Each kind of tree derived from the intermediate graph and the combination of these 3 trees should also be evaluated by the same way as the intermediate graph evaluation to check the coverage of them to capture the SLG.

As shown in Table 5, we provide the evaluation results of the derived trees (individuals and combination) on CROHME 2014 test set at the symbol level. Tree-Time catch $75.54 \%$ ground truth spatial relationships and $23.16 \%$ ( false positive/targets) unnecessary ones. The evaluation results of Tree-Left-R1 is very close to Tree-0-R1 since the first input stoke is the leftmost stroke for many cases. They capture around $68 \%$ ground truth spatial relationships and around $70 \%$ unnecessary ones. When we combine these 3 trees, a better ground truth relationships coverage, $92.17 \%$, is achieved with the unnecessary ones of $60.50 \%$. Compared to the intermediate graph, the combination of these 3 derived trees catches slightly less ground truth relationships but greatly less unnecessary relationships which verify the effectiveness of the method for deriving trees.

Table 5 The derived trees evaluation results at the symbol level on CROHME 2014 test set (provided the ground truth labels of nodes and edges)

\begin{tabular}{|c|cc|cc|cc|}
\hline Model & \multicolumn{2}{|c|}{ Segments (\%) } & \multicolumn{2}{c|}{ Seg + Class (\%) } & \multicolumn{2}{c|}{ Tree Rels. (\%) } \\
\hline & Rec. & Prec. & Rec. & Prec. & Rec. & Prec. \\
\hline Tree-Time & 99.73 & 99.45 & 99.72 & 99.44 & 75.54 & 76.54 \\
\hline Tree-Left-R1 & 94.07 & 87.85 & 94.06 & 87.85 & 68.86 & 49.91 \\
\hline Tree-0-R1 & 94.51 & 88.16 & 94.50 & 88.15 & 67.98 & 49.03 \\
\hline Merge3 & 99.84 & 98.74 & 99.83 & 98.73 & 92.17 & 60.37 \\
\hline
\end{tabular}

\subsubsection{Classifiers evaluation}

In this section we now label the nodes and edges of derived trees with the BLSTM classifier. The evaluation results on symbol level and global expression 
Table 6 The symbol level evaluation results on CROHME 2014 test set with 3 trees, including the experiment results in this work and CROHME 2014 participant results

\begin{tabular}{|c|cc|cc|cc|}
\hline System & \multicolumn{2}{|c|}{ Segments (\%) } & \multicolumn{2}{|c|}{ Seg + Class (\%) } & \multicolumn{2}{c|}{ Tree Rels. (\%) } \\
\hline & Rec. & Prec. & Rec. & Prec. & Rec. & Prec. \\
\hline i, Tree-Time & 92.93 & 84.82 & 84.12 & 76.78 & 60.70 & 76.19 \\
\hline i, Tree-Left-R1 & 84.82 & 72.49 & 72.80 & 62.21 & 44.34 & 57.78 \\
\hline i, Tree-0-R1 & 85.31 & 72.88 & 74.17 & 63.37 & 42.92 & 60.08 \\
\hline i, Merge3 & $\mathbf{9 3 . 5 3}$ & $\mathbf{8 7 . 2 0}$ & $\mathbf{8 6 . 1 0}$ & $\mathbf{8 0 . 2 8}$ & $\mathbf{7 1 . 1 6}$ & $\mathbf{6 6 . 1 3}$ \\
\hline ii, Tree-Time & 95.10 & 90.47 & 87.53 & 83.27 & 65.06 & 83.18 \\
\hline ii, Tree-Left-R1 & 86.71 & 75.64 & 76.85 & 67.03 & 48.14 & 61.91 \\
\hline ii, Tree-0-R1 & 87.52 & 76.66 & 77.00 & 67.45 & 48.14 & 63.04 \\
\hline ii, Merge3 & $\mathbf{9 5 . 0 1}$ & $\mathbf{9 0 . 0 5}$ & $\mathbf{8 8 . 3 8}$ & $\mathbf{8 3 . 7 6}$ & $\mathbf{7 6 . 2 0}$ & $\mathbf{7 2 . 2 8}$ \\
\hline iii, Tree-Time & 95.43 & 91.13 & 88.26 & 84.28 & 65.45 & 83.57 \\
\hline iii, Tree-Left-R1 & 88.03 & 78.13 & 78.56 & 69.72 & 50.31 & 65.87 \\
\hline iii, Tree-0-R1 & 87.41 & 77.02 & 77.63 & 68.40 & 48.23 & 64.28 \\
\hline iii, Merge3 & $\mathbf{9 5 . 2 5}$ & $\mathbf{9 0 . 7 0}$ & $\mathbf{8 8 . 9 0}$ & $\mathbf{8 4 . 6 5}$ & $\mathbf{7 7 . 3 3}$ & $\mathbf{7 3 . 7 2}$ \\
\hline \hline Merge 9 & $\mathbf{9 5 . 5 2}$ & $\mathbf{9 1 . 3 1}$ & $\mathbf{8 9 . 5 5}$ & $\mathbf{8 5 . 6 0}$ & $\mathbf{7 8 . 0 8}$ & $\mathbf{7 4 . 6 4}$ \\
\hline \hline & \multicolumn{7}{|c|}{ CROHME 2014} & participant & results & \\
\hline MyScript & 98.42 & 98.13 & 93.91 & 93.63 & 94.26 & 94.01 \\
\hline València & 93.31 & 90.72 & 86.59 & 84.18 & 84.23 & 81.96 \\
\hline Nantes & 89.43 & 86.13 & 76.53 & 73.71 & 71.77 & 71.65 \\
\hline RIT-CIS & 88.23 & 84.20 & 78.45 & 74.87 & 61.38 & 72.70 \\
\hline RIT-DRPL & 85.52 & 86.09 & 76.64 & 77.15 & 70.78 & 71.51 \\
\hline Tokyo & 83.05 & 85.36 & 69.72 & 71.66 & 66.83 & 74.81 \\
\hline São Paulo & 76.63 & 80.28 & 66.97 & 70.16 & 60.31 & 63.74 \\
\hline
\end{tabular}

Table 7 The expression level evaluation results on CROHME 2014 test set with 3 trees, including the experiment results in this work and CROHME 2014 participant results

\begin{tabular}{|c|c|c|c|c|}
\hline System & correct (\%) & $\leq 1$ error & $\leq 2$ errors & $\leq 3$ errors \\
\hline i, Tree-Time & 12.41 & 20.24 & 26.14 & 30.93 \\
\hline i, Tree-Left-R1 & 5.9 & 10.58 & 15.99 & 19.94 \\
\hline i, Tree-0-R1 & 5.39 & 10.47 & 16.28 & 20.14 \\
\hline i, Merge3 & $\mathbf{1 9 . 9 4}$ & $\mathbf{2 7 . 5 7}$ & $\mathbf{3 3 . 8 8}$ & $\mathbf{3 9 . 3 7}$ \\
\hline ii, Tree-Time & 16.09 & 25.46 & 32.28 & 37.27 \\
\hline ii, Tree-Left-R1 & 6.82 & 13.33 & 20.14 & 23.19 \\
\hline ii, Tree-0-R1 & 6.41 & 13.02 & 18.41 & 23.40 \\
\hline ii, Merge3 & $\mathbf{2 5 . 9 4}$ & $\mathbf{3 6 . 7 2}$ & $\mathbf{4 2 . 3 2}$ & $\mathbf{4 6 . 5 9}$ \\
\hline iii, Tree-Time & 16.80 & 25.56 & 32.89 & 38.09 \\
\hline iii, Tree-Left-R1 & 8.55 & 15.26 & 20.96 & 24.52 \\
\hline iii, Tree-0-R1 & 7.93 & 13.63 & 19.63 & 25.43 \\
\hline iii, Merge3 & $\mathbf{2 9 . 3 0}$ & $\mathbf{3 9 . 0 6}$ & $\mathbf{4 3 . 6 4}$ & $\mathbf{4 8 . 0 2}$ \\
\hline \hline Merge 9 & $\mathbf{2 9 . 9 1}$ & $\mathbf{3 9 . 9 4}$ & $\mathbf{4 4 . 9 6}$ & $\mathbf{5 0 . 1 5}$ \\
\hline \hline \multicolumn{5}{|c|}{ CROHME 2014} \\
\hline MyScript & 62.68 & 72.31 & 75.15 & 76.88 \\
\hline València & 37.22 & 44.22 & 47.26 & 50.20 \\
\hline Nantes & 26.06 & 33.87 & 38.54 & 39.96 \\
\hline Tokyo & 25.66 & 33.16 & 35.90 & 37.32 \\
\hline RIT-DRPL & 18.97 & 28.19 & 32.35 & 33.37 \\
\hline RIT-CIS & 18.97 & 26.37 & 30.83 & 32.96 \\
\hline São Paulo & 15.01 & 22.31 & 26.57 & 27.69 \\
\hline
\end{tabular}

level are presented in Tables 6 and 7 respectively. We give both the individual tree recognition results and the merging results in each table. Tree-Time covers all the strokes of the input expression but can miss some relational edges between strokes; Tree-Left-R1 and Tree-0-R1 could catch some additional edges which are not covered by Tree-Time. The experiment results also verified this tendency. Compared to (iii, Tree-Time), the symbol segmentation 
and classification results of (iii, Merge3) stay at almost the same level while the recall rate of relationship classification is greatly improved (about 12\%). The different recognition results of network (ii) are systematically increased when compared to (i) as the deep structure could get higher level representations of the input data. The performance of network (iii) is moderately improved when compared to (ii), just as same as the case in Experiment 1. When we consider merging all these 9 classifiers, we also get a slight improvement as shown by Merge 9.

We compare the results of Merge 9 to the systems in CROHME 2014. With regard to the symbol classification and recognition rates, our system performs better than the second-ranked system in CROHME 2014. For relationship classification rate, our system reaches the level between the second-ranked and the third-ranked systems in CROHME 2014. The global expression recognition rate is $29.91 \%$, ranking third in all the participated systems. When we compute the recognition rate with $\leq 3$ errors, our result is $50.15 \%$, very close to the second-ranked system $(50.20 \%)$.

The top ranked system MyScript is built on the principle that segmentation, recognition and interpretation have to be handled concurrently and at the same level in order to result in the best candidate. They use a much larger training data set which is not available to the public. System València parses expressions using two-dimensional stochastic context-free grammars. It is an advanced model evolution of the system presented in [34]. System Nantes simultaneously optimizes expression segmentation, symbol recognition, and 2-D structure recognition under the restriction of an expression grammar [33]. The approach transforms the recognition problem into a search for the best possible interpretation of a sequence of input strokes. Furthermore, all the top 4 systems in the CROHME 2014 competition are grammar driven solutions which need a large amount of manual work and a high computational complexity. There is no grammar considered in our system.

We also evaluate the system of Merge 9 on CROHME 2016 test data set (Tables 8 and 9 ). As can be seen in Table 8, compared to other participated

Table 8 The symbol level evaluation results on CROHME 2016 test set with the system of Merge 9, along with CROHME 2016 participant results

\begin{tabular}{|c|cc|cc|cc|}
\hline System & \multicolumn{2}{|c|}{ Segments (\%) } & \multicolumn{2}{|c|}{ Seg + Class (\%) } & \multicolumn{2}{c|}{ Tree Rels. (\%) } \\
\hline & Rec. & Prec. & Rec. & Prec. & Rec. & Prec. \\
\hline \hline Merge 9 & 95.64 & 91.44 & 89.84 & 85.90 & 77.23 & 74.08 \\
\hline & \multicolumn{7}{|c|}{ CROHME 2016 participant } & results \\
\hline MyScript & 98.89 & 98.95 & 95.47 & 95.53 & 95.11 & 95.11 \\
\hline Wiris & 96.49 & 97.09 & 90.75 & 91.31 & 90.17 & 90.79 \\
\hline Tokyo & 91.62 & 93.25 & 86.05 & 87.58 & 82.11 & 83.64 \\
\hline São Paulo & 92.91 & 95.01 & 86.31 & 88.26 & 81.48 & 84.16 \\
\hline Nantes & 94.45 & 89.29 & 87.19 & 82.42 & 73.20 & 68.72 \\
\hline
\end{tabular}

systems in CROHME 2016, our system is still competitive on symbol segmentation and classification task. For relationship recognition task, there is room 
Table 9 The expression level evaluation results on CROHME 2016 test set with the system of Merge 9, along with CROHME 2016 participant results

\begin{tabular}{|c|c|c|c|}
\hline System & correct (\%) & $\leq 1$ error & $\leq 2$ errors \\
\hline Merge 9 & 27.03 & 35.48 & 42.46 \\
\hline & CROHME 2016 participant results \\
\hline MyScript & 67.65 & 75.59 & 79.86 \\
\hline Wiris & 49.61 & 60.42 & 64.69 \\
\hline Tokyo & 43.94 & 50.91 & 53.70 \\
\hline São Paulo & 33.39 & 43.50 & 49.17 \\
\hline Nantes & 13.34 & 21.02 & 28.33 \\
\hline
\end{tabular}

for improvement. The results at expression level are presented in Table 9. The global expression recognition rate is $27.03 \%$.

\subsection{Error analysis}

In this section, we make a deep error analysis of the recognition result of (Merge 9 ) to better understand the system and to explore the directions for improving recognition rate in future. The Label Graph Evaluation library (LgEval) [55] evaluates the recognition system by comparing the output SLG of each expression with its ground truth SLG. Thus, node label confusion matrix and edge label confusion matrix are available. Based on the two confusion matrices, we analyze the errors specifically below.

Node label. In table 10, we list the types of SLG node label error which have a high frequency on CROHME 2014 test set recognized by (Merge 9) system. The first column gives the outputted node labels by the classifier; the second column provide the ground truth node labels, along with the number of nodes with each label; the last column records the corresponding number of occurrences, also the percentages. As can be seen from the table, the most frequent error $(x \rightarrow X, 46)$ belongs to the type of the lowercase-uppercase errors. Moreover, $(p \rightarrow P, 24),(c \rightarrow C, 16),(X \rightarrow x, 16)$ and $(y \rightarrow Y, 14)$ also belong to the same type of lowercase-uppercase errors. Another type of error which happens quite often in our experiment is the similar-look error, such as $(x \rightarrow \times, 26),(\times \rightarrow x, 10),(z \rightarrow 2,10),(q \rightarrow 9,10)$ and so on. With more training samples, we can expect a better discrimination of these similar classes. Another improvement would be to integrate explicitly a language model to promote frequent symbols.

Edge label. Table 11 provides the edge (SLG) label errors of CROHME 2014 test set using (Merge 9). As can be seen, a large amount of errors come from the last row which represents the missing edges. 1858 edges with label Right are missed in our system, along with 929 segmentation edges. In addition, we can see the errors of high frequency in the sixth row which represents that five relationship (exclude Right) edges or segmentation edges or NoRelation (_) edges are mis-classified as Right edges. Among them, 1600 NoRelation (-) edges are recognized as Right. The post process step of Make connected SRT is one of the reasons since we take a hard decision (add Right edges) in this step. Another possible reason is that, as Right relationship is 
Table 10 Illustration of node (SLG) label errors of (Merge 9) on CROHME 2014 test set. We only list the cases that occur at least 10 times

\begin{tabular}{|c|c|c|}
\hline output label & $\begin{array}{c}\text { ground truth label } \\
\text { (no. of nodes with this label) }\end{array}$ & $\begin{array}{c}\text { no. of occurrences } \\
\text { (percentage) }\end{array}$ \\
\hline $\mathrm{x}$ & $\mathrm{X}(60)$ & $46(76.7 \%=46 / 60)$ \\
\hline $\mathrm{x}$ & $\times(145)$ & $26(17.93 \%)$ \\
\hline $\mathrm{P}$ & $\mathrm{P}(30)$ & $24(80 \%)$ \\
\hline, & $1(794)$ & $19(2.39 \%)$ \\
\hline $\mathrm{c}$ & $\mathrm{C}(31)$ & $16(51.61 \%)$ \\
\hline $\mathrm{y}$ & $\mathrm{Y}(26)$ & $14(53.85 \%)$ \\
\hline+ & $\mathrm{t}(162)$ & $14(8.64 \%)$ \\
\hline$\cdot$ & $\ldots(123)$ & $13(10.57 \%)$ \\
\hline $\mathrm{X}$ & $\mathrm{x}(890)$ & $16(1.80 \%)$ \\
\hline $\mathrm{a}$ & $\mathrm{x}(890)$ & $14(1.57 \%)$ \\
\hline 1 & $1(62)$ & $11(17.74 \%)$ \\
\hline- & $1(794)$ & $10(1.26 \%)$ \\
\hline $\mathrm{x}$ & $\mathrm{x}(890)$ & $10(1.12 \%)$ \\
\hline $\mathrm{z}$ & $2(713)$ & $10(1.40 \%)$ \\
\hline $\mathrm{q}$ & $9(98)$ & $10(10.20 \%)$ \\
\hline
\end{tabular}

the most frequent relation in math expressions, the classifiers may answer too often this frequent class.

We explore deeper the problem of the missing edges which appear in the last row of Table 11. In fact, there exist three sources which result in the missing

Table 11 Illustration of edge (SLG) label errors of (Merge 9) on CROHME 2014 test set. The first column represents the output labels; the first row offers the ground truth labels, as well as the the number of edges with each label; other cells in this table provide the corresponding no. of occurrences. '*' represents segmentation edges, grouping two nodes into a symbol. The label of segmentation edge is a symbol(For convenient representation, we do not give the specific symbol types, but an overall label '*'.)

\begin{tabular}{|c|c|c|c|c|c|c|c|c|}
\hline & $*(9044)$ & Above (592) & Below (627) & Inside (377) & Right (13698) & Sub (1115) & Sup (923) & $-(261528)$ \\
\hline$*$ & 208 & 0 & 0 & & 17 & 1 & 1 & 29 \\
\hline Above & 8 & & 1 & & & & 21 & 10 \\
\hline Below & 2 & & & 1 & 1 & 114 & & 7 \\
\hline Inside & & 5 & 1 & & & 1 & & 9 \\
\hline Right & 344 & 65 & 22 & 40 & & 152 & 112 & 1600 \\
\hline Sub & 4 & & 6 & 3 & 44 & & 1 & 7 \\
\hline Sup & 1 & 3 & & & 35 & 3 & & 31 \\
\hline- & 929 & 300 & 80 & 109 & 1858 & 189 & 235 & \\
\hline
\end{tabular}

edges: (1) the edges are missed at the graph representation stage. We evaluated the graph model in Section 5.2.1 where around 6\% relationships were missed. One of the future works could be searching for a better graph representation model to catch the $6 \%$ missing relationships. (2) Some edges in the derived graph are recognized by the system as NoRelation (_), which actually have a ground truth label of one of 6 relationship or symbol (segmentation edge). (3) Even if we derive multiple trees from the graph $G$, they do not well cover the graph completely. Thus, a better strategy for deriving trees from the graph will be explored in future works. 
We illustrate one test sample $\left(\frac{9}{9+\sqrt{9}}\right)$ from CROHME 2014 test set recognized by system (Merge9). We provide the handwritten input, along with the built SLG for this test sample (Figure 16). For this expression, the structure of it was correctly recognized, only one error being the first symbol ' 9 ' of the denominator was recognized as ' $\rightarrow$ '. This error belongs to the type of the similar-look error we have explained in error analysis section. Enlarging the training data set could be a solution to solve it. Also, it could be eased by introducing a language model since $\frac{9}{\rightarrow+\sqrt{9}}$ is not a valid expression from this point of view.

\section{Conclusion}

In this work, we developed a tree-BLSTM based recognition system for online handwritten mathematical expression. To be able to process 2-D languages, we extend the chain-structured BLSTM to tree-based BLSTM which could model the dependencies in a tree structure, and extend CTC to local CTC to relatively constrain the outputs and at the same time benefit from introducing an additional 'blank' class. One major difference with the traditional approaches is that there is no explicit segmentation, recognition and layout extraction steps but a unique trainable system that produces directly a SLG describing a mathematical expression. The proposed system, without using any grammar, achieves competitive results in online math expression recognition domain.

Based on the current method and error analysis, we summarize several possible directions for future work. Some work should be done with regards to improve the existing method, like improving the graph model, proposing a better strategy for deriving trees and developing a stronger post process stage. Some efforts could be put into introducing language model into the graph. For example, as known an n-gram model is widely used in 1-D language processing like text and speech, how to take into account the statistical properties of ngrams in math expression recognition task is an interesting direction to explore in future. Another interesting work could be to extend BLSTM model to a DAG structure which will better cover the derived graph and therefore be able to handle more contextual information compared to the tree structure BLSTM. The current recognition system achieves competitive results without using any grammar knowledge. In future, we could apply graph grammar to improve the current recognition rate. In this paper, we extend the chain-structured BLSTM to a tree topology to let it model the dependency directly in a tree structure. Furthermore, we extend the CTC training technique to local CTC to constrain the output position relatively at the same time improve the system training efficiency compared to frame-wise training. These proposed algorithms are generic ones and could be apply in other research fields such as diagram or flowchart recognition.

Acknowledgements We would like to thank the China Scholarship Council (CSC) for supporting PhD studentship at Université de Nantes. 


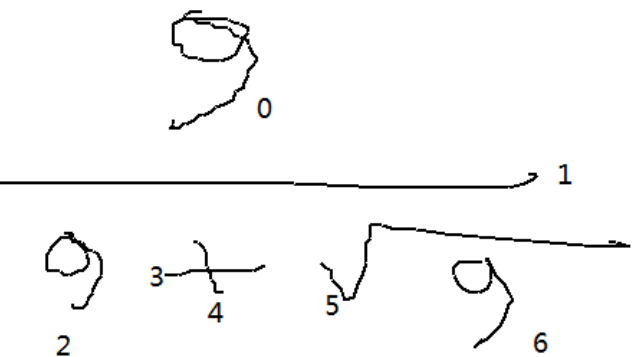

(a)

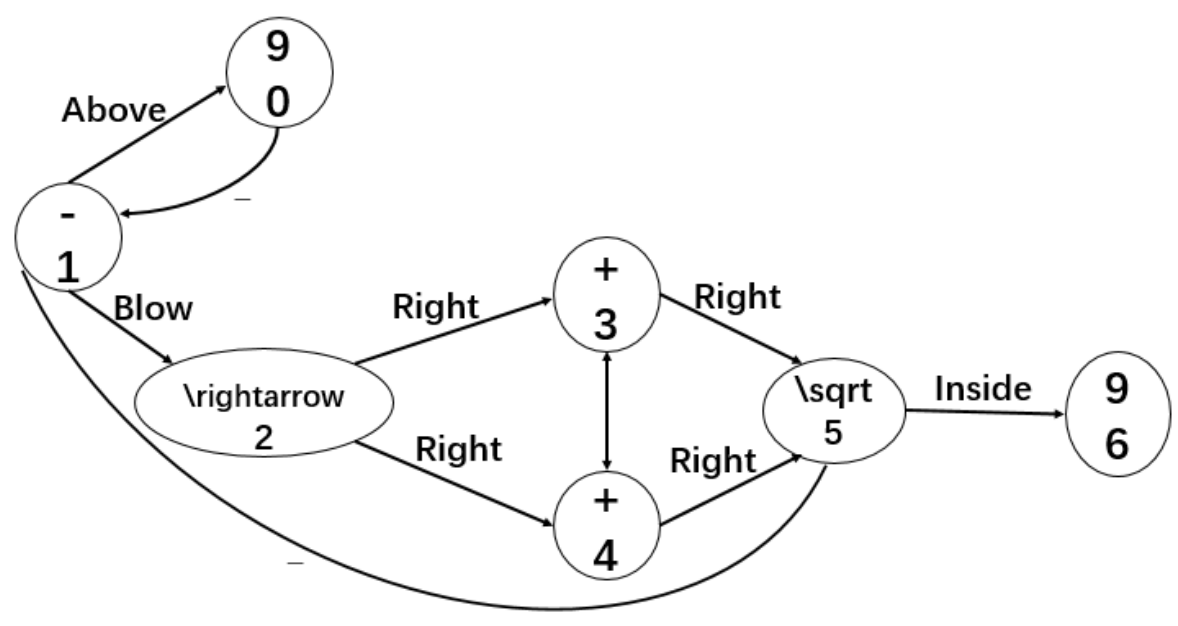

(b)

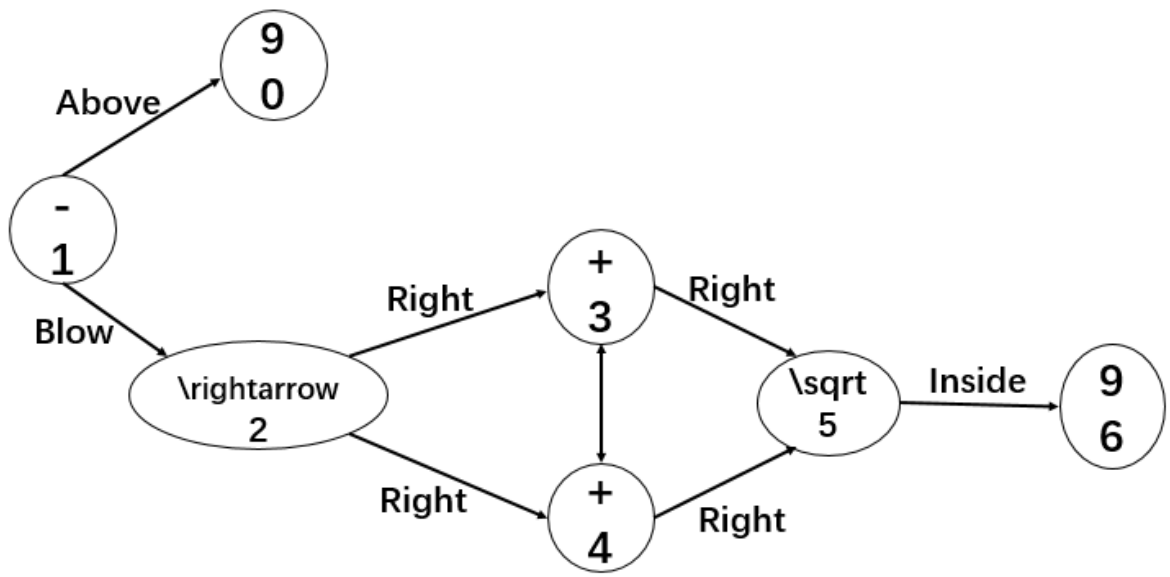

(c)

Fig. 16 (a) $\frac{9}{9+\sqrt{9}}$ written with 7 strokes; (b)the SLG after merging several trees and performing other post processing steps; (c)the SLG with NoRelation edges removed. There is a node label error: the stroke 2 with the ground truth label ' 9 ' was wrongly classified as ' $\rightarrow$ ' 


\section{Compliance with Ethical Standards}

All authors declare that: (1) no support, financial or otherwise, has been received from any organization that may have an interest in the submitted work ; and (2) there are no other relationships or activities that could appear to have influenced the submitted work."

\section{References}

1. Kremer R (1988) Visual languages for knowledge representation. 11th Workshop on Knowledge Acquisition, Modeling and Management

2. Marriott K, Meyer B, Wittenburg KB (1998) A Survey of Visual Language Specification and Recognition. In: Marriott K, Meyer B (eds) Visual Language Theory. Springer, New York, 5-85

3. Zanibbi R, Blostein D (2012) Recognition and retrieval of mathematical expressions. International Journal on Document Analysis and Recognition (IJDAR), 15(4): 331-357

4. Mouchère H, Zanibbi R, Garain U, Viard-Gaudin C (2016) Advancing the state of the art for handwritten math recognition: the CROHME competitions, 2011-2014. International Journal on Document Analysis and Recognition (IJDAR), 19(2): 173-189

5. Graves A, Liwicki M, Fernández S, Bertolami R, Bunke H, Schmidhuber, J (2009) A novel connectionist system for unconstrained handwriting recognition. IEEE Transactions on Pattern Analysis and Machine Intelligence, 31(5): 855-868

6. Álvaro Muñoz F, Sánchez JA, Benedí JM (2013) Classification of on-line mathematical symbols with hybrid features and recurrent neural networks. 12th International Conference on Document Analysis and Recognition (ICDAR), 1012-1016

7. Zhang H, Li J, Ji Y, Yue H (2017) Understanding Subtitles by Character-Level Sequenceto-Sequence Learning. IEEE Transactions on Industrial Informatics, 13(2): 616-624

8. Tai KS, Socher R, Manning CD (2015) Improved semantic representations from treestructured long short-term memory networks. arXiv:1503.00075

9. Zhu XD, Sobhani P, Guo HY (2015) Long Short-Term Memory Over Recursive Structures. International Conference on Machine Learning(ICML), 1604-1612

10. Zhu XD, Sobhani P, Guo HY (2016) Dag-structured long short-term memory for semantic compositionality. Proceedings of NAACL-HLT, 917-926

11. Zhang T, Mouchère H, Viard-Gaudin C (2016) Online Handwritten Mathematical Expressions Recognition by Merging Multiple 1D Interpretations. 15th International Conference on Frontiers in Handwriting Recognition (ICFHR), 187-192

12. Zhang T, Mouchère H, Viard-Gaudin C (2016) Using BLSTM for interpretation of 2-D languages. Document numérique, Lavoisier, 19(2): 135-157

13. Anderson RH (1967) Syntax-directed recognition of hand-printed two-dimensional mathematics. Symposium on Interactive Systems for Experimental Applied Mathematics, 436-459

14. Chang SK (1970) A method for the structural analysis of two-dimensional mathematical expressions. Information Sciences, 2(3): 253-272

15. Martin WA (1971) Computer input/output of mathematical expressions. Proceedings of the second ACM symposium on Symbolic and algebraic manipulation, 78-89

16. Anderson RH (1977) Two-dimensional mathematical notation. Syntactic Pattern Recognition, Applications, 147-177

17. Blostein D, Grbavec A (1997) Recognition of mathematical notation. Handbook of character recognition and document image analysis, 21: 557-582

18. Chan KF, Yeung DY (2000) Mathematical expression recognition: a survey. International Journal on Document Analysis and Recognition, 3(1): 3-15

19. Tapia E, Rojas R (2007) A survey on recognition of on-line handwritten mathematical notation. Tech report, Free University of Berlin, Department of Computer Science

20. Chou PA (1989) Recognition of equations using a two-dimensional stochastic contextfree grammar. Visual Communications and Image Processing IV, 1199: 852-863 
21. Koschinski M, Winkler HJ, Lang M (1995) Segmentation and recognition of symbols within handwritten mathematical expressions. International Conference on Acoustics, Speech, and Signal Processing(ICASSP), 2439-2442

22. Winkler HJ, Fahrner H, Lang M (1995) A soft-decision approach for structural analysis of handwritten mathematical expressions. International Conference on Acoustics, Speech, and Signal Processing(ICASSP), 2459-2462

23. Lehmberg S, Winkler HJ, Lang M (1996) A soft-decision approach for symbol segmentation within handwritten mathematical expressions. IEEE International Conference on Acoustics, Speech, and Signal Processing(ICASSP), 3434-3437

24. Matsakis NE (1999) Recognition of handwritten mathematical expressions. PhD thesis, Massachusetts Institute of Technology

25. Zanibbi R, Blostein D, Cordy JR (2002) Recognizing mathematical expressions using tree transformation. IEEE Transactions on pattern analysis and machine intelligence, 24(11): 1455-1467

26. Tapia E, Rojas R (2003) Recognition of on-line handwritten mathematical expressions using a minimum spanning tree construction and symbol dominance. International Workshop on Graphics Recognition, 329-340

27. Toyozumi K, Yamada N, Kitasaka T, Mori K, Suenaga Y, Mase K, Takahashi T (2004) A study of symbol segmentation method for handwritten mathematical formula recognition using mathematical structure information. 17th International Conference on Pattern Recognition(ICPR), 630-633

28. Tapia E (2005) Understanding mathematics: A system for the recognition of on-line handwritten mathematical expressions. PhD thesis, Freie Universität Berlin

29. Zhang L, Blostein D, Zanibbi R (2005) Using fuzzy logic to analyze superscript and subscript relations in handwritten mathematical expressions. Eighth International Conference on Document Analysis and Recognition, 972-976

30. Shi Y, Li HY, Soong FK (2007) A unified framework for symbol segmentation and recognition of handwritten mathematical expressions. Ninth International Conference on Document Analysis and Recognition(ICDAR), 854-858

31. Aly W, Uchida S, Fujiyoshi A, Suzuki M (2009) Statistical classification of spatial relationships among mathematical symbols. 10th International Conference on Document Analysis and Recognition(ICDAR), 1350-1354

32. Yamamoto R, Sako S, Nishimoto T, Sagayama S (2006) On-line recognition of handwritten mathematical expressions based on stroke-based stochastic context-free grammar. Tenth International Workshop on Frontiers in Handwriting Recognition, 249-254

33. Awal AM, Mouchère H, Viard-Gaudin C (2014) A global learning approach for an online handwritten mathematical expression recognition system. Pattern Recognition Letters, 35(1): $68-77$

34. Álvaro Muñoz F, Sánchez JA, Benedí JM (2014) Recognition of on-line handwritten mathematical expressions using $2 \mathrm{~d}$ stochastic context-free grammars and hidden markov models. Pattern Recognition Letters,35(1): 58-67

35. Álvaro Muñoz F, Sánchez JA, Benedí JM (2016) An integrated grammar-based approach for mathematical expression recognition. Pattern Recognition, 51: 135-147

36. MacLean S, Labahn G (2013) A new approach for recognizing handwritten mathematics using relational grammars and fuzzy sets. International Journal on Document Analysis and Recognition (IJDAR), 16(2): 139-163

37. Celik M, Yanikoglu B (2011) Probabilistic mathematical formula recognition using a 2d context-free graph grammar. International Conference on Document Analysis and Recognition (ICDAR), 161-166

38. Julca-Aguilar F (2016) Recognition of Online Handwritten Mathematical Expressions using Contextual Information. PhD thesis, Université de Nantes, Université Bretagne Loire, Universidade de São Paulo

39. Deng YT, Kanervisto A, Rush AM (2016) What You Get Is What You See: A Visual Markup Decompiler. arXiv:1609.04938

40. Zhang JS, Du J, Zhang SL, Liu D, Hu YL, Hu JS, Wei S, Dai LR (2017) Watch, Attend and Parse: An End-to-end Neural Network Based Approach to Handwritten Mathematical Expression Recognition. Pattern Recognition, 71: 196-206 
41. Zanibbi R, Mouchère H, Viard-Gaudin C (2013) Evaluating structural pattern recognition for handwritten math via primitive label graphs. Proc. SPIE 8658, Document Recognition and Retrieval XX, 865817

42. Graves A (2012) Supervised sequence labelling with recurrent neural networks. Springer 43. Hochreiter S, Bengio Y, Frasconi P, Schmidhuber J (2001) Gradient flow in recurrent nets: the difficulty of learning long-term dependencies. IEEE Press

44. Hochreiter S, Schmidhuber J (1997) Long short-term memory. Neural computation, 9(8): 1735-1780

45. Graves A, Schmidhuber J (2005) Framewise phoneme classification with bidirectional LSTM networks. 2005 IEEE International Joint Conference on Neural Networks (IJCNN), 2047-2052

46. Graves A, Jaitly N, Mohamed AR (2013) Hybrid speech recognition with deep bidirectional LSTM. IEEE Workshop on Automatic Speech Recognition and Understanding (ASRU), 273-278

47. Graves A, Schmidhuber J (2009) Offline handwriting recognition with multidimensional recurrent neural networks. Advances in neural information processing systems, 545-552

48. Messina R, Louradour J (2015) Segmentation-free handwritten Chinese text recognition with LSTM-RNN. 13th International Conference on Document Analysis and Recognition (ICDAR), 171-175

49. Bluche T, Louradour J, Messina R (2016) Scan, attend and read: End-to-end handwritten paragraph recognition with mdlstm attention. arXiv:1604.03286

50. Maalej R, Kherallah M (2016) Improving MDLSTM for Offline Arabic Handwriting Recognition Using Dropout at Different Positions. International Conference on Artificial Neural Networks, 431-438

51. Maalej R, Tagougui N, Kherallah M (2016) Recognition of handwritten Arabic words with dropout applied in MDLSTM. International Conference Image Analysis and Recognition, 746-752

52. Álvaro Muñoz F (2015) Mathematical Expression Recognition based on Probabilistic Grammars. PhD thesis, Universitat Politècnica de València

53. Bluche T, Ney H, Louradour J, Kermorvant C (2015) Framewise and CTC training of Neural Networks for handwriting recognition. 13th International Conference on Document Analysis and Recognition (ICDAR), 81-85

54. Álvaro Muñoz F, Sánchez JA, Benedí JM (2014) Offline Features for Classifying Handwritten Math Symbols with Recurrent Neural Networks. 2014 22nd International Conference on Pattern Recognition (ICPR), 2944-2949

55. Mouchère H, Viard-Gaudin C, Zanibbi R, Garain U (2014) Crohme 2014: competition on recognition of on-line handwritten mathematical expressions. 2014 14th International Conference on Frontiers in Handwriting Recognition (ICFHR), 791-796

56. $\mathrm{Hu} \mathrm{L} \mathrm{(2016)} \mathrm{Features} \mathrm{and} \mathrm{Algorithms} \mathrm{for} \mathrm{Visual} \mathrm{Parsing} \mathrm{of} \mathrm{Handwritten} \mathrm{Mathematical}$ Expressions. PhD thesis, Rochester Institute of Technology 